This work is licensed under a Creative Commons Attribution 3.0 License.

\author{
Research article
}

urn:1sid:zoobank.org:pub:A7ED0BAC-2637-433D-9BD2-8A9E2C59B2CA

\title{
Revision of the genus Spilopteron Townes, 1965 (Hymenoptera: Ichneumonidae: Acaenitinae) from Japan
}

\author{
Masato ITO ${ }^{1, *} \&$ Kaoru MAETO ${ }^{2}$ \\ ${ }^{1,2}$ Laboratory of Insect Biodiversity and Ecosystem Science, Graduate School of Agricultural Science, \\ Kobe University, Rokkodai-cho 1-1, Nada-ku, Kobe, Hyogo 657-8501, Japan. \\ *Corresponding author: fixsenia@hotmail.co.jp \\ ${ }^{2}$ Email: maeto@kobe-u.ac.jp \\ ${ }^{1}$ urn:Isid:zoobank.org:author:86EEEA2C-F825-4C72-A7F9-F4A324B2C045 \\ ${ }^{2}$ urn:lsid:zoobank.org:author:BEBD5A8E-EC49-42CF-8E1E-C375287A0B36
}

\begin{abstract}
Ten Japanese species of the genus Spilopteron Townes, 1965 are recognized. Five new species, S. albiventre sp. nov., S. brachyurum sp. nov., S. nigrum sp. nov., S. oblongulum sp. nov. and S. pseudonigrum sp. nov., are described from Japan. Morphological discrimination between most Japanese species is confirmed by sequence analysis of the mitochondrial COI gene, which indicates the following relationships: S. oblongulum sp. nov. + S. apicale (Matsumura, 1912), S. brachyurum sp. nov. + S. nigrum sp. nov. + S. pseudonigrum sp. nov., and S. tosaense (Uchida, 1934) + S. luteum (Uchida, 1930). A key to the Japanese species of Spilopteron is provided. This genus seems to have its center of diversity in the mid-latitude area of East Asia.
\end{abstract}

Keywords. New species, parasitoid, mtCOI, taxonomy, geographical color variation, melanism, East Asia.

Ito M. \& Maeto K. 2017. Revision of the genus Spilopteron Townes, 1965 (Hymenoptera: Ichneumonidae: Acaenitinae) from Japan. European Journal of Taxonomy 356: 1-33. https://doi.org/10.5852/ejt.2017.356

\section{Introduction}

Spilopteron Townes, 1965 is a medium-sized genus of the ichneumonid subfamily Acaenitinae, containing 30 species, of which eleven are known from the Palearctic region, four from the Nearctic region, and 15 from the Oriental region (Yu et al. 2012). Host insects reported for Spilopteron species are as follows: Pygoleptura nigrella (Say, 1826) and Strangalepta vittata (Olivier, 1792) (Cerambycidae) for S. vicinum (Cresson, 1869) (Cushman \& Rohwer 1920; Townes et al. 1960), Strangalia bicolor (Swederus, 1787) (Cerambycidae) for S. occiputale (Cresson, 1869) (Cushman \& Rohwer 1920; Townes et al. 1960), Bellamira scalaris (Say, 1826) (Cerambycidae) for S. formosum (Cresson, 1868) (Champlain 1922; Townes 1944), Pyrrhona laeticolor (Bates, 1884) (Cerambycidae) for S. pyrrhonae Kusigemati, 1981, and Eriotremex formosanus (Matsumura, 1912) (Siricidae) for S. fuscomaculatum Wang, 1988 (Sheng \& Sun 2010); the non-coleopteran host record (E. formosanus) should be considered doubtful. Shaw \& Wahl (1989) reported that a related species Acaenitus dubitator (Panzer, 1800) is a 
koinobiont endoparasitoid of curculionid larvae, and thus species of Spilopteron may also have the same strategy of parasitism.

In Japan, five species of Spilopteron have hitherto been recorded: S. apicale (Matsumura, 1912), S. tosaense (Uchida, 1934), S. pyrrhonae, S. mucronatus Lee, 2008, and S. luteum (Uchida, 1930), (Kusigemati 1981; Ito et al. 2012).

The purpose of this study is to revise the five established Japanese species of Spilopteron and to propose five new species, based on morphological and molecular evidence. We also provide a key to the Japanese species of Spilopteron.

\section{Material and methods}

\section{Morphological examination}

Specimens from the following collections were used: the Department of Systematic Entomology, Hokkaido University, Sapporo (SEHU) (M. Ohara); the National Institute of Agro-Environmental Science, Tsukuba (NIAES) (S. Yoshimatsu); the National Museum of Nature and Science, Tsukuba (NSMT) (A. Shinohara); the Kanagawa Prefectural Museum of Natural History, Odawara (KPMNH) (K. Watanabe); the Entomological Laboratory of Meijo University, Nagoya (MU) (K. Yamagishi); the Osaka Museum of Natural History, Osaka (OMNH) (R. Matsumoto); the Entomological Laboratory of Kagoshima University, Kagoshima (KU) (Y. Sakamaki).

Specimens were observed using a stereoscopic microscope (Nikon SMZ660) and an optical microscope (Nikon ECLIPSE 50i). Images were taken using a digital microscope (Keyence Digital Microscope VHX-600) (Figs 1-2, 4-5) or a scanning electron microscope (JEOL JSM-6010LV) (Figs 3, 6). General morphological terminology follows Gauld $(1991,2002)$, and terminology for surface sculpture follows Eady (1968). The following abbreviations are used in descriptions: metasomal tergite (T), and metasomal sternite (S). The anterior, raised section of the pronotum is indicated as the "collar".

\section{Mitochondrial DNA analysis}

Partial DNA sequences of the mtCOI gene, so-called DNA barcodes, are widely used for the taxonomy of Hymenoptera (e.g., Sakurai et al. 2009; Stigenberg et al. 2011). Those of all Japanese species, except for $S$. albiventre sp. nov., were analyzed with Yamatarotes chishimensis (Uchida, 1929) (GenBank accession number AB932961 (Ito \& Maeto 2014)) as an outgroup. The accession numbers of specimens are indicated in square brackets ([ ] ) under "Material examined".

Adult specimens were preserved in $99.5 \%$ ethanol and stored at $-30^{\circ} \mathrm{C}$ until dissection. A middle tarsus was removed and preserved in $99.5 \%$ ethanol. After drying, the tarsus was ground in $20 \mu 1$ of $50 \mathrm{mM}$ $\mathrm{NaOH}$ and digested for $15 \mathrm{~min}$ at $95^{\circ} \mathrm{C}$. Thereafter, the sample was neutralized with $20 \mu 1$ of $200 \mathrm{mM}$ Tris-HCl.

The mtCOI primers designed by Folmer et al. (1994) (LCO1490: 5'-GGT CAA CAA ATC ATA AAG ATA TTG G-3'; HCO2198: 5'-TAA ACT TCA GGG TGA CCA AAA AAT CA-3') (648 bp) were used for the polymerase chain reaction (PCR), which was conducted with KOD FX NEO (Toyobo). Cycling conditions were $94^{\circ} \mathrm{C}$ for $2 \mathrm{~min}$, followed by 35 cycles at $98^{\circ} \mathrm{C}$ for $10 \mathrm{~s}, 48^{\circ} \mathrm{C}$ for $30 \mathrm{~s}$, and $68^{\circ} \mathrm{C}$ for $15 \mathrm{~s}$. The PCR products were purified using the illustra GFX kit (GE Healthcare Life Sciences). Gene regions were sequenced using the same primers as used in the PCRs with the BigDyeTM Terminator ver. 3.1 Cycle Sequencing kit (Applied Biosystems). Cycling conditions were 25 cycles at $96^{\circ} \mathrm{C}$ for $10 \mathrm{~s}, 50^{\circ} \mathrm{C}$ for $5 \mathrm{~s}$, and $60^{\circ} \mathrm{C}$ for $4 \mathrm{~min}$. The products were purified using $3 \mathrm{M}$ sodium acetate, $95 \%$ ethanol, and $70 \%$ 
ethanol, and redissolved by the Hi-Di Formamide (Applied Biosystems). Cycle sequencing reactions were run on the ABI Prism 3100 Genetic Analyzer (Applied Biosystems).

Sequence data were assembled using the DNA Dynamo Sequence Analysis Software (Blue Tractor Software) and were aligned using ClustalW (http://www.genome.jp/tools/clustalw/) to obtain 590 alignable base pairs for analysis. Genetic distances were calculated according to the Kimura's twoparameter model (Kimura 1980), implemented in MEGA6 (Tamura et al. 2013). Maximum likelihood (ML) trees were constructed using MEGA6 with 1000 bootstrap replications (Tamura et al. 2013). The model selection for ML was performed according to the Akaike information criterion (AIC) in MEGA6. The best-fit substitution model was Kimura's two-parameter distance, with rate heterogeneity among sites modeled using a proportion of a gamma distribution $(+\mathrm{G})$. All the DNA sequences obtained are deposited in the DDBJ (http://www.ddbj.nig.ac.jp/index-j.html)/EMBL GenBank database (https:// www.ncbi.nlm.nih.gov/genbank/).

\section{Results}

\section{Analysis of mitochondrial DNA}

Our ML analysis of mtCOI sequences demonstrated nine compact clusters of Japanese Spilopteron (except for $S$. albiventre sp. nov., the sequence of which was not examined because the specimens were too old), within each of which the genetic distances among specimens were less than $3 \%$ in Kimura's two-parameter model (Fig. 9). They all corresponded exactly to the five already known and four new species described below. The analysis indicated the following relationships: $S$. oblongulum sp. nov. + S. apicale (bootstrap value: 95, mean genetic distance: $7.5 \%$ ), S. brachyurum sp. nov. + S. nigrum sp. nov. + S. pseudonigrum sp. nov. (bootstrap value: 81 , mean genetic distance: $9.7 \%$ ), and $S$. tosaense + S. luteum (bootstrap value: 68, mean genetic distance: $10.9 \%$ ).

\section{Taxonomy}

Class Insecta Linnaeus, 1758

Order Hymenoptera Linnaeus, 1758

Family Ichneumonidae Latreille, 1802

Subfamily Acaenitinae Förster, 1869

Genus Spilopteron Townes, 1960

Spilopteron Townes, 1960 in Townes \& Townes 1960: 568. Original designation. Type-species: Spilopteron franclemonti Townes, 1960.

\section{Diagnosis}

Head 0.6-0.7 times as long as wide in dorsal view. Face strongly punctate and weakly convex (Fig. 2). Clypeus with a transverse median ridge (Figs 2-3). Frons punctate, with a median longitudinal carina (Fig. 2). Subocular groove present (Fig. 2). Mandible striate except apex (Figs 2-3). Lower tooth of mandible longer than upper tooth. Vertex and gena weakly and sparsely punctate. Occiput smooth. Occipital carina complete. Scutellum roundly convex in lateral view. Propodeum roundly convex in lateral view (Fig. 5). Propodeal spiracle elongate (Fig. 5). Fore wing with areolet open (Fig. 4). Hind wing with vein $C u \& c u-a$ intercepted near middle (Fig. 4). Hind tarsus claws without an accessory tooth. Basal convexity of S1 covered with some erect setae. Ovipositor tip without transverse ridges.

\section{Distribution}

Palearctic, Oriental and Nearctic regions. 


\section{Systematic position}

The genus used to belong to the tribe Acaenitini sensu Townes (1971), but Wahl \& Gauld (1998) proposed the discontinuance of the use of tribes within the subfamily Acaenitinae; we follow their opinion here. This genus closely resembles Siphimedia Cameron, 1902, known from the Oriental region, and Metachorischizus Uchida, 1928 from the Palearctic and Oriental regions, but it can be distinguished from them by the maxillary palpus with short hairs (vs long hairs in Siphimedia), the propodeal spiracle not enclosed by an elliptical carina (vs enclosed in Siphimedia), the propodeum with distinct regular carinae (vs irregular carinae in Metachorischizus), and the bullae in $2 m-c u$ a little distant from each other (vs very close in Metachorischizus).

\section{Spilopteron albiventre sp. nov. urn:1sid:zoobank.org:act:5EAE1212-248A-4020-8684-0FCE656C56A9}

Figs 1a-b, 2a, 3a, 4a, 5a, 6a, 7a-b, 8a

\section{Etymology}

This specific epithet of this new species is derived from the yellowish white metasomal apex.

\section{Type material}

\section{Holotype}

JAPAN: , "Hyakumatsu-zawa, Sapporo-shi, Hokkaido, Japan, 20. July 2001, T. Yoshida leg." (SEHU).

\section{Paratypes}

JAPAN: $2+q$, same locality as holotype, Malaise trap, 12-18 Jul. 2001, T. Yoshida (SEHU); 1 , same locality as holotype, Malaise trap, 4-18 Aug. 2001, T. Yoshida (SEHU); 1 q, Kannon-zawa, Sapporpshi, Hokkaido, Malaise trap, 24 Aug.-7 Sep. 1993, N. Kuhara (NIAES); 1 + , same locality, Malaise trap, 1-8 Aug. 2000, K. Uesugi (KPMNH); 1 +, Mt Soranuma-dake, Sapporo-shi, Hokkaido, Malaise trap, 27 Jul.-21 Aug. 2007, A. Ueda (OMNH); 1 , Onneto, Ashoro-cho, Hokkaido, Malaise trap, 23 Jul.-30 Aug. 2002, K. Uesugi (KPMNH); 1 §, Nippu, Bifuka-cho, Hokkaido, Malaise trap, 19 Jun.-17 Jul. 2010, N. Kuhara and M. Sueyoshi (OMNH); 1 Jै, Mt Hachimori-yama, Asahi-mura, Nagano Pref., 25 Jul. 1998, T. Tachi (OMNH); 1 đ̊, Maruno-cho, Nirasaki-shi, Yamanashi Pref., 26 Jun.-20 Jul. 2007, K. Hosoda (OMNH); 8 đ̋̃, Mt Odamiyama, Ehime Pref., 27 Jun. 1997, E. Yamamoto (NIAES); 1 q, same locality, 10 Jun. 1997, E. Yamamoto (NIAES); 1 q, same locality, 1 Jul. 1995, E. Yamamoto (NIAES); Onogahara, Ehime Pref., 6-7 Jul. 1979, Y. Seiyama (EUM).

\section{Differential diagnosis}

The new species most resembles S. brachyurum sp. nov. and S. pyrrhonae, but it is distinguished from them by the long malar space, 1.3-1.4 times as long as the basal mandibular width (0.9-1.2 times in S. brachyurum and S. pyrrhonae), and the yellowish white marking on the apex of the metasoma (entirely black in S. brachyurum and S. pyrrhonae).

\section{Description}

Female $(\mathrm{n}=11)$

Body LeNGTH. 7.5-10.0 mm.

HEAD. Vertex 0.4-0.5 times as long as maximum length of eye in dorsal view. Frons without a depression between eye and antennal socket in frontal view (Fig. 2a). Clypeus 0.5-0.7 times as long as wide, with three weak projections of equal size (Figs $2 \mathrm{a}, 3 \mathrm{a}$ ). Face $0.7-0.8$ times as long as wide. Length of malar space 1.3-1.4 times as long as basal mandibular width. Ocello-ocular line/lateral ocellar diameter 


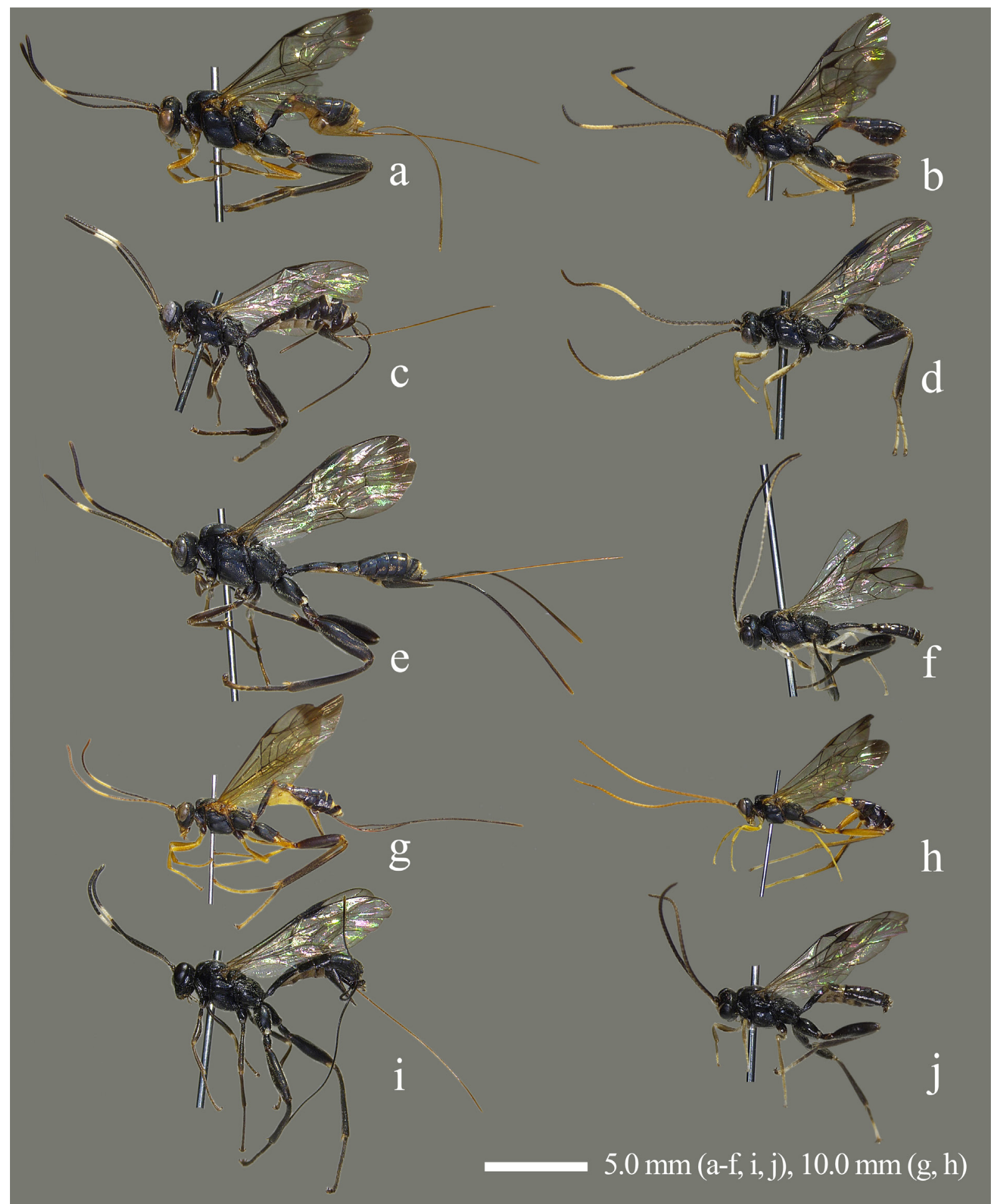

Fig. 1. Habitus in lateral view. a-b. Spilopteron albiventre sp. nov. a. Holotype,, , Ehime Pref. b. Paratype, $\widehat{\jmath}$, Ehime Pref. - c-d. Spilopteron brachyurum sp. nov. c. Holotype, + , Hokkaido. d. Paratype, $\widehat{\partial}$, Hokkaido. - e-f. Spilopteron nigrum sp. nov. e. Holotype,, , Wakayama Pref. f. Paratype, ${ }^{\lambda}$, Hyogo Pref. g-h. Spilopteron oblongulum sp. nov. g. Holotype,, , Kanagawa Pref. h. Paratype, $\widehat{\partial}$, Hyogo Pref. i-j. Spilopteron pseudonigrum sp. nov. i. Holotype,, , Hyogo Pref. j. Paratype, $\widehat{\jmath}$, Hyogo Pref.. 
$=1.1-1.3$. Postocellar line/lateral ocellar diameter $=0.7-1.0$. Antenna with 30-31 flagellomeres; first flagellomere 1.3-1.5 times as long as second flagellomere.

Mesosoma. Propleuron densely punctate (Fig. 5a). Lateral area of pronotum weakly punctate and transversely striate medially. Collar sparsely punctate. Mesoscutum densely punctate (Fig. 5a). Subalar prominence densely punctate (Fig. 5a). Scutellum weakly punctate in dorsal view. Postscutellum flattened in lateral view (Fig. 5a). Metapleuron strongly punctate (Fig. 5a). Propodeum indistinctly carinate (Fig. 6a). Area superomedia confluent with area petiolaris. Fore wing length $6.5-9.0 \mathrm{~mm}$. Vein $c u-a$ basal to vein $R s \& M$ (Fig. 4a). Vein $r s-m$ opposite or basal to vein $2 m-c u$ (Fig. 4a). Hind femur 3.2-3.5 times as long as maximum depth in lateral view, without distinct convexity ventrally. Hind tibia $10.0-11.7$ times as long as maximum depth in lateral view. First hind tarsomere 2.1-2.6 times as long as second and 1.9-2.2 times as long as longer hind tibial spur.

Metasoma. T1 1.9-2.2 times as long as maximum width, 1.9-2.2 times as long as T2. T2 0.7 times as long as maximum width. T1 sparsely punctate. T2-T8 densely punctate. S1 without distinct sharp projection basally. Ovipositor sheath $2.1-2.5$ times as long as hind tibia.

Color. Body black (Fig. 1a). Antennal flagellum with a white band (Fig. 1a). Inner margin of eye yellowish white, pale color sometimes extended to most of face (Fig. 2a). Clypeus, basal area of mandible, scutellum, postscutellum, propodeum, apices of metasomal tergites, apex of metasoma, and subgenital plate yellowish white (propodeum and T1 sometimes entirely black). Fore and mid legs yellowish brown (Fig. 1a). Fore wing with a rounded apical dark mark (Fig. 4a).

Male $(\mathrm{n}=12$; genitalia, $\mathrm{n}=2)$

Similar to female (Fig. 1b). Body length $6.0-10.0 \mathrm{~mm}$. Head $0.6-0.7$ times as long as wide in dorsal view. Vertex $0.4-0.5$ times as long as maximum length of eye in dorsal view. Ocello-ocular line/ lateral ocellar diameter $=1.0-1.4$. Postocellar line/lateral ocellar diameter $=0.8-1.2$. Antenna with $31-35$ flagellomeres. Hind femur 3.4-3.6 times as long as maximum depth in lateral view. Hind tibia 10.0-11.7 times as long as maximum depth in lateral view. First hind tarsomere 1.9-2.2 times as long as longer hind tibial spur. T1 2.0-2.5 times as long as maximum width, 1.8-2.1 times as long as T2. T2 0.7-0.8 times as long as maximum width. Face entirely yellow.

Subgenital plate pentagonal with a basal angle obtuse (Fig. 8a). Paramere short, basal part strongly projected toward the base of subgenital plate (Fig. 7a). Aedeagus gently curved, its penis valve ca 2.0 times as long as basal apodeme (Fig. 7b).

\section{Distribution}

Japan (Hokkaido, Honshu, and Shikoku).

\section{Bionomics}

Host unknown. Adults fly mainly in July and August.

Spilopteron apicale (Matsumura, 1912)

Figs $2 b, 3 b, 4 b, 5 b, 6 b, 7 c-d, 8 b$

Chorischizus apicalis Matsumura, 1912: 149. Type locality: Japan, Hokkaido, Sapporo City.

Phaenolobus apicalis - Uchida 1928: 34.

Arotes apicalis - Uchida 1932: 160.

Siphimedia apicalis - Uchida 1934: 52. 
Spilopteron apicalis - Townes et al. 1965: 391. — Kusigemati 1981: 121 (in part).

Spilopteron apicale - Yu et al. 2005, 2012. — Ito et al. 2015: 546.

\section{Material examined}

JAPAN: 1 q (lectotype of Chorischizus apicalis), Jozan, Sapporo-shi, Hokkaido, late Aug. 1907, S. Matsumura (SEHU); 1 \& (paralectotype of Chorischizus apicalis), Sapporo-shi, Hokkaido, Aug. 1907, S. Matsumura (SEHU); 1 + , same locality as lectotype, early Sep. 1914, S. Matsumura (SEHU); 1 , same locality as lectotype, 18 Aug. 1915, S. Matsumura (SEHU); 2 우, same locality as paralectotype, 17 Sep. 1932, C. Watanabe (SEHU); 1 ㅇ, Maruyama, Sappori-shi, Hokkaido, 21 Sep. 1931, M. Takizawa (SEHU); 1 q, Hitsujigaoka, Sappori-shi, Hokkaido, 1-8 Aug. 2011, K. Konishi (LC041250 OMNH); 8 우, Soranumadake, Sapporo-shi, Hokkaido, 27 Jul.-21 Aug. 2007, A. Ueda (OMNH, EUM); 1 , , same locality, 4-27 Jul. 2007, A. Ueda (OMNH); 2 우, Kuriyama-cho, Yubari-

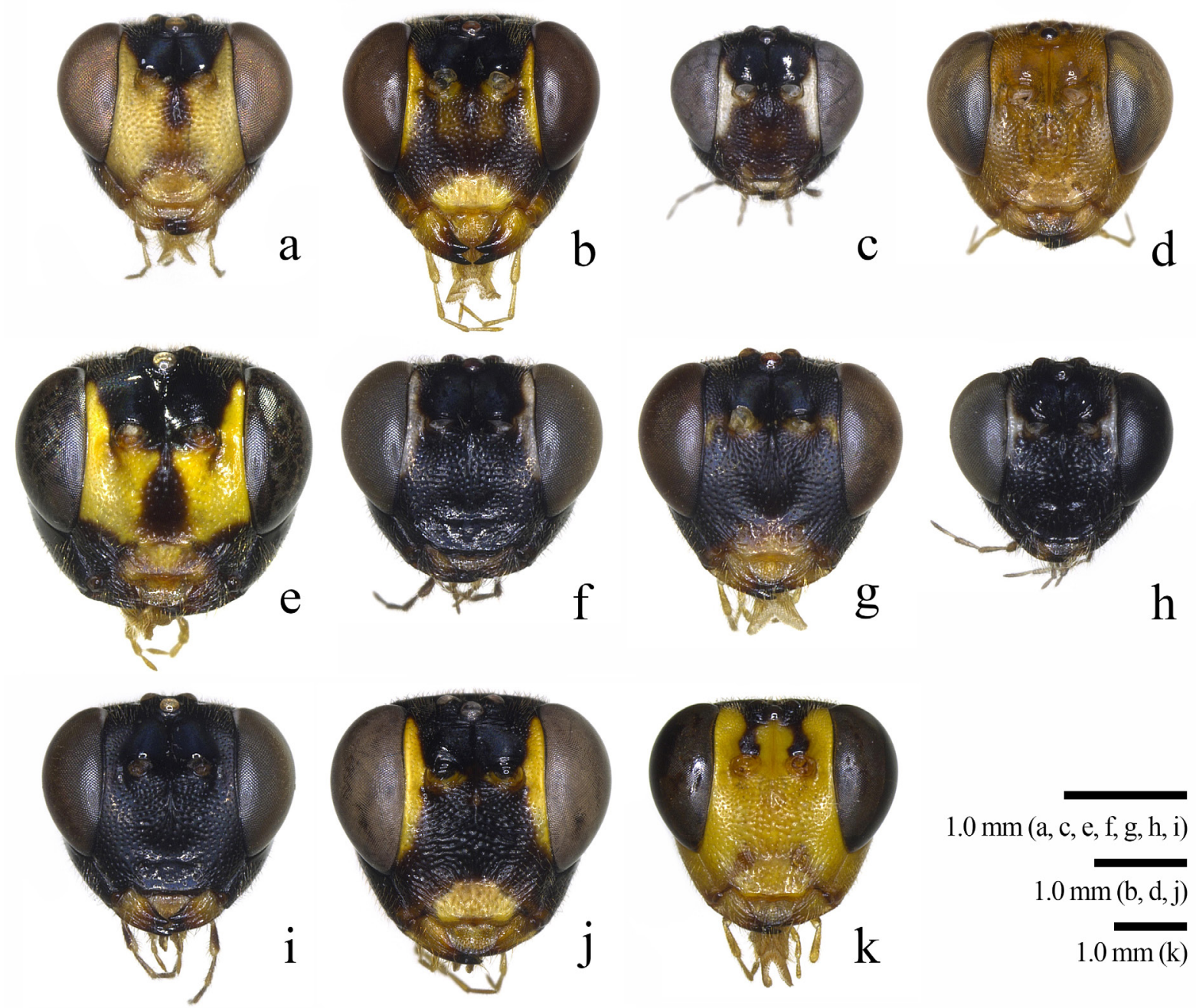

Fig. 2. Head of females in frontal view. a. Spilopteron albiventre sp. nov., paratype, Ehime Pref. b. Spilopteron apicale (Matsumura, 1912), Nagano Pref. c. Spilopteron brachyurum sp. nov., paratype, Hokkaido. d. Spilopteron luteum (Uchida, 1930), Amami-oshima Is. e. Spilopteron mucronatus Lee, 2008, Fukuoka Pref. f. Spilopteron nigrum sp. nov., paratype, Hyogo Pref. g. Spilopteron oblongulum sp. nov., paratype, Kanagawa Pref. h. Spilopteron pseudonigrum sp. nov., paratype, Hyogo Pref. i. Spilopteron pyrrhonae Kusigemati, 1981, Kanagawa Pref. j. Spilopteron tosaense (Uchida, 1934), Hokkaido. k. Spilopteron tosaense (Uchida, 1934), Yakushima Is. 
shi, Hokkaido, 24 Jul.- 24 Aug. 2006, A. Ueda (OMNH); 1 \&, Yubari-shi, Hokkaido, 3-17 Aug. 2007, A. Ueda (OMNH); 2 q $q$, same locality, 24 Jul.-24 Aug. 2006, A. Ueda (OMNH); 1 q, Takinoshita, Kuriyama-cho, Hokkaido, 16 Jul.-5 Aug. 2009, A. Ueda (OMNH); 2 우, Chikkabetsu, Tokachi, Hokkaido, 29-31 Jul. 1946, Takahashi, Kosugi and Sakagami (SEHU); 6 우, Tomuraushi, Hokkaido, 24-27 Aug. 1954, C. Watanabe et al. (SEHU); 3 우, Kushiro, Hokkaido, 20 Aug. 1942, T. Uchida (SEHU); 1 \&, Kawayu, Kushiro, Hokkaido, 1 Sep. 1941, T. Uchida (SEHU); 1 q, same locality, 8 Aug. 1947, S. Sakagami (SEHU); 1 +, Niibushi, Kushiro, Hokkaido, 9 Aug. 1947, S. Sakagami (SEHU); 1 ○, Kamioboro, Kushiro, Hokkaido, 6 Aug. 1947, S. Sakagami (SEHU); 1 \&, Utoro-Iwaobetsu, Shiretoko, Hokkaido, 15-17 Aug. 1953, T. Kumata (SEHU); 1 , Teshio, Hokkaido, 6 Sep. 1956, K. Kamijo (SEHU); 1 ㅇ, Kawaratai, Nishimeya-mura, Aomori Pref., 6-14 Aug. 2013, T. Nakamura (EUM); 1 , Tsukuba Univ., Sugadaira-kogen, Ueda-shi, Nagano Pref., 8 Aug.-3 Sep. 2014, S. Shimizu (KPMNH); 1 , same locality, 3-26 Sep. 2014, S. Shimizu (KPMNH); 3 + 9 , Utsukushimatsu, Daimon, Nagawacho, Nagano Pref., 25 Aug. 2011, S. Fujie (LC041254, LC041255 OMNH); 2 우, same locality, 22 Aug. 2012, S. Fujie (LC041256 OMNH); 1 \&, same locality, 23 Aug. 2012, S. Fujie (LC041257 OMNH); 1 , same locality, 26 Aug. 2011, S. Fujie (OMNH); 1 , same locality, 27 Aug. 2011, S. Fujie $(\mathrm{OMNH}) ; 7$ 우, Ontake-kogen, Outaki-mura, Nagano Pref., 31 Jul. 2013, M. Ito (KPMNH); 2 우으,

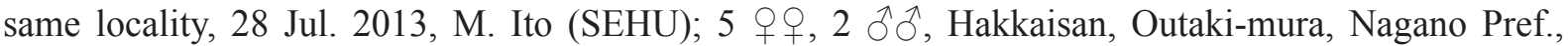

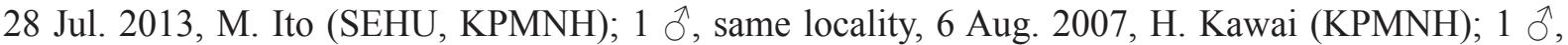
same locality, 7 Aug. 2010, K. Watanabe (KPMNH); 2 우, 1 ô, Karei-kogen, Ina-shi, Nagano Pref., 30 Jul. 2013, K. Watanabe (KPMNH); 1 , same locality, 30 Jul. 2013, N. Kikuchi (KPMNH); 1 , Karasugawa-keikoku, Hotakamaki, Azumino-shi, Nagano Pref., 24 Aug. 2012, S. Fujie (OMNH); 1 ð’, Mt Asamayama, Nagano Pref., 1 Aug. 1978, K. Mizuno (OMNH); 1 q, Biwakubosawa, Masutomi, Hokuto-shi, Yamanashi Pref., 7 Aug. 2007, K. Watanabe (OMNH); 1 , same locality, 8 Aug. 2008, K. Watanabe (OMNH); 3 우, same locality, 28 Jul.-7 Aug. 2007, K. Watanabe (OMNH); 1 , same locality, 8 Aug. 2008, K. Watanabe, (OMNH); 1 , , Hikawa, Enzan-shi, Yamanashi Pref., 5 Aug. 2008, H. Katahira (KPMNH); 1 ภे, Tano, Yamato-cho, Koshu-shi, Yamanashi Pref., 5 Aug. 2008, K. Watanabe (KPMNH); 1 §̊, Shorakuji, Akeno-mura, Yamanashi Pref., 7 Aug. 2007, K. Watanabe (KPMNH); 5 우, Nabedaira-kogen, Takayama-shi, Gifu Pref., 13 Aug. 2013, M. Ito (NSMT); 1 , same locality, 3 Aug. 2013, S. Fujie (NIAES); 1 + , 1 ô, Kute, Nyukawa-cho, Takayama-shi, Gifu Pref., 4 Aug. 2013, S. Fujie (NIAES); 1 ㅇ, Odaigahara, Kamikitayama-mura, Nara Pref., 15-22 Aug. 2005, A. Kawazoe (OMNH); 1 , Sobosan, Oita Pref., 28 Jul. 1978, R. Noda (NIAES).

\section{Differential diagnosis}

Until the revision by Ito et al. (2015), this species had been confused with $S$. tosaense due to color variation. However, it can be distinguished from $S$. tosaense by the following character states: fore wing with the apical dark mark rounded and not extending downward (extending downward in S. tosaense), and without a dark mark below the pterostigma (Fig. 4b); frons with a depression between eye and antennal socket (absent in $S$. tosaense); and clypeus with a very weak median projection and two strong lateral projections (Fig. 3b) (with three equal-sized projections in S. tosaense).

\section{Description \\ Female $(\mathrm{n}=84)$ \\ BODY LENGTH. 13.5-16.0 mm.}

Head. Vertex 0.6-0.7 times as long as maximum length of eye in dorsal view. Frons with a depression between eye and antennal socket in frontal view (Fig. 2b). Clypeus $0.6-0.7$ times as long as wide, with two strong lateral projections and a weak median projection (Figs $2 b, 3 b$ ). Face 0.6 times as long as wide. Length of malar space 0.9-1.2 times as long as basal mandibular width. Ocello-ocular line/lateral ocellar diameter $=1.6-2.1$. Postocellar line/lateral ocellar diameter $=1.0-1.4$. Antenna with 34-35 flagellomeres; first flagellomere 1.2 times as long as second flagellomere. 
Mesosoma. Propleuron densely punctate (Fig. 5b). Lateral area of pronotum densely punctate and transversely striate medially (Fig. 5b). Collar sparsely punctate. Mesoscutum densely punctate. Subalar prominence extensively punctate (Fig. 5b). Scutellum sparsely punctate in dorsal view. Postscutellum roundly convex in lateral view (Fig. 5b). Metapleuron strongly and densely punctate (Fig. 5b). Propodeum strongly carinate (Fig. 6b). Area externa weakly punctate (Fig. 6b). Area basalis weakly carinate (Fig. 6b). Area dentipara, area posteroexterna and area petiolaris confluent with area superomedia, and weakly carinate (Fig. 6b). Fore wing length $10.5-14.0 \mathrm{~mm}$. Vein $c u-a$ basad of vein $R s \& M$ (Fig. $4 \mathrm{~b}$ ). Vein $r s-m$ opposite or distad of vein $2 m-c u$ (Fig. $4 \mathrm{~b}$ ). Hind femur 4.1-4.2 times as long as maximum depth in lateral view, without distinct convexity ventrally. Hind tibia $11.4-12.7$ times as long as maximum depth in lateral view. First hind tarsomere 2.5-2.6 times as long as second one and 3.3-3.6 times as long as longer hind tibial spur.

Metasoma. T1 2.2-2.6 times as long as maximum width, 2.2-2.6 times as long as T2. T2 0.7 times as long as maximum width. T1 weakly and sparsely punctate. T2-T8 weakly and densely punctate. S1 without distinct sharp projection basally. Ovipositor sheath 1.8 times as long as hind tibia.

CoLor. Body black (Fig. 5b). Antennal flagellum with a white band. Inner margin of eye, clypeus, basal area of mandible, subalar prominence, scutellum, postscutellum, apex of propodeum, fore and mid legs, hind trochanter, hind trochantellus, apex of hind tarsus, and apices of T1 and T2 yellowish brown. Fore

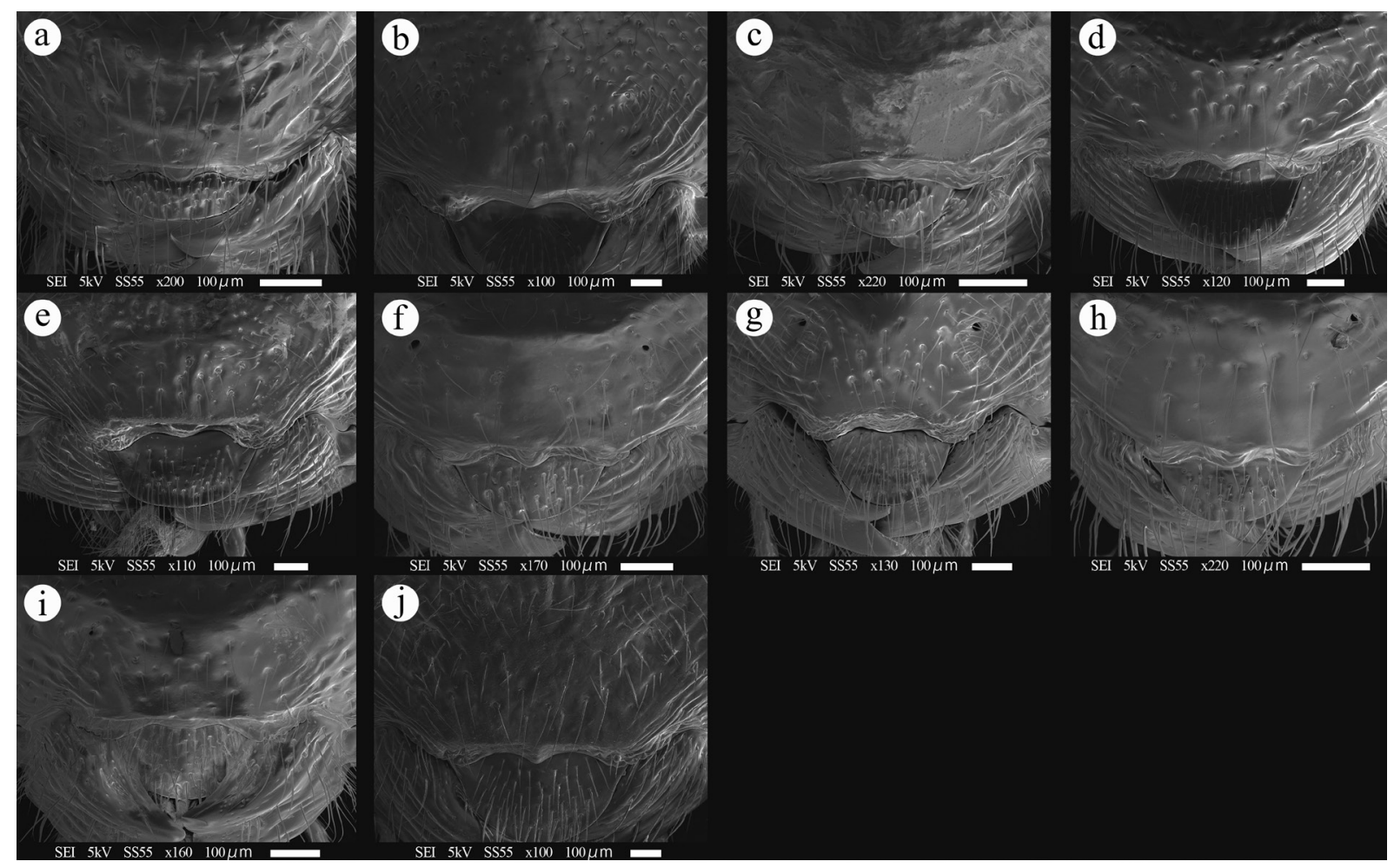

Fig. 3. Clypeus of female in frontal view. a. Spilopteron albiventre sp. nov., paratype, Ehime Pref. b. Spilopteron apicale (Matsumura, 1912), Nagano Pref. c. Spilopteron brachyurum sp. nov., paratype, Hokkaido. d. Spilopteron luteum (Uchida, 1930), Amami-oshima Is. e. Spilopteron mucronatus Lee, 2008, Fukuoka Pref. f. Spilopteron nigrum sp. nov., paratype, Hyogo Pref. g. Spilopteron oblongulum sp. nov., paratype, Kanagawa Pref. h. Spilopteron pseudonigrum sp. nov., paratype, Hyogo Pref. i. Spilopteron pyrrhonae Kusigemati, 1981, Kanagawa Pref. j. Spilopteron tosaense (Uchida, 1934), Hokkaido. 
wing with an apical dark mark rounded and not extending downward, without a dark mark below the pterostigma (Fig. 4b).

Male ( $\mathrm{n}=10$; genitalia, $\mathrm{n}=3$ )

Similar to female. Body length $12.5-15.0 \mathrm{~mm}$. Head 0.6 times as long as wide. Face 0.7 times as long as wide. Length of malar space $0.8-1.1$ times as long as basal mandibular width. Ocello-ocular line/ lateral ocellar diameter $=1.3-1.5$. Postocellar line/lateral ocellar diameter $=1.2-1.4$. Antenna with $38-$ 39 flagellomeres; first flagellomere 1.1-1.4 times as long as second flagellomere. Hind tibia 11.7-13.2 times as long as maximum depth in lateral view. First hind tarsomere 2.1-2.3 times as long as second and 3.6-4.8 times as long as longer hind tibial spur. T1 2.4-3.3 times as long as maximum width, 2.02.4 times as long as T2. T2 $0.8-0.9$ times as long as maximum width. Face entirely yellow. Antennal flagellum without a white band.

Subgenital plate hexagonal with an obtuse basal angle (Fig. 8b). Paramere short, basal part strongly projecting towards base of subgenital plate (Fig. 7c). Aedeagus gently curved, its penis valve ca 2.0 times as long as basal apodeme (Fig. 7d).

\section{Distribution}

Japan (Hokkaido, Honshu, Shikoku, and Kyushu), China, and Far East Russia (Sakhalin Oblast).

\section{Bionomics}

Host unknown. In Honshu MI often observed females ovipositing into trunks of Japanese larch (Larix kaempferi (Lamb.) Carr.) (Pinaceae), into which a Cerambycidae (Etorofus (Nakanea) vicaria (Bates, 1844)) was also ovipositing. Adults fly in July and August, visiting flowers of Angelica pubescens Maxim. (Apiaceae) and Aralia cordata Thunb. (Araliaceae).

\section{Spilopteron brachyurum sp. nov. urn:1sid:zoobank.org:act:FDA2ACF6-526E-479C-BF27-0B0B8DB1B0C9}

Figs $1 \mathrm{c}-\mathrm{d}, 2 \mathrm{c}, 3 \mathrm{c}, 4 \mathrm{c}, 5 \mathrm{c}, 6 \mathrm{c}, 7 \mathrm{e}-\mathrm{f}, 8 \mathrm{c}$

\section{Etymology}

The specific epithet of this new species is derived from the short ovipositor sheath.

\section{Type material}

\section{Holotype}

JAPAN: + , “Mt. tarumae-san, Hokkaido, Japan, 12-18 July 1998, K. Konishi leg. (MsT)” (NIAES).

\section{Paratypes}

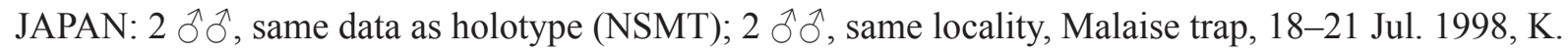

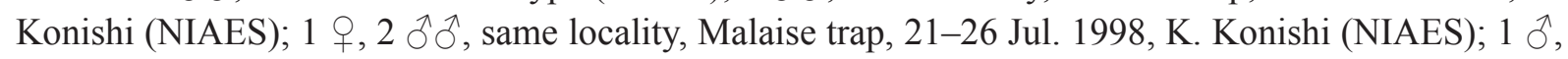
Mt Teinekanayama, Sapporo-shi, Hokkaido, 19 Jul. 2012, M. Ito (LC154925 OMNH); 1 Oे, Nanamagari, Aomori-shi, Aomori Pref., 25 Jul.-1 Aug. 1992, T. Ichita (NIAES).

\section{Differential diagnosis}

This new species most resembles $S$. albiventre sp. nov. and S. pyrrhonae, but it is easily distinguished from both by the rounded area superomedia on the propodeum (the area is square in $S$. albiventre and S. pyrrhonae). 


\section{Description}

Female $(\mathrm{n}=2)$

BODY LENGTH. 6.5-8.0 mm

HEAD. Vertex 0.4-0.6 times as long as maximum length of eye in dorsal view. Frons without a depression between eye and antennal socket in frontal view (Fig. 2c). Clypeus 0.4-0.5 times as long as wide, with a median projection (Figs 2c, 3c). Face 0.6 times as long as wide. Length of malar space 0.9-1.0 times as long as basal mandibular width. Ocello-ocular line/lateral ocellar diameter $=1.2-1.5$. Postocellar line/ lateral ocellar diameter $=1.0$. Antenna with 29 flagellomeres; first flagellomere 1.2-1.3 times as long as second flagellomere.

Mesosoma. Propleuron densely punctate (Fig. 5c). Lateral area of pronotum densely punctate and transversely striate medially. Collar weakly punctate. Mesoscutum densely punctate (Fig. 5c). Subalar prominence sparsely punctate (Fig. 5c). Scutellum densely punctate in dorsal view. Postscutellum

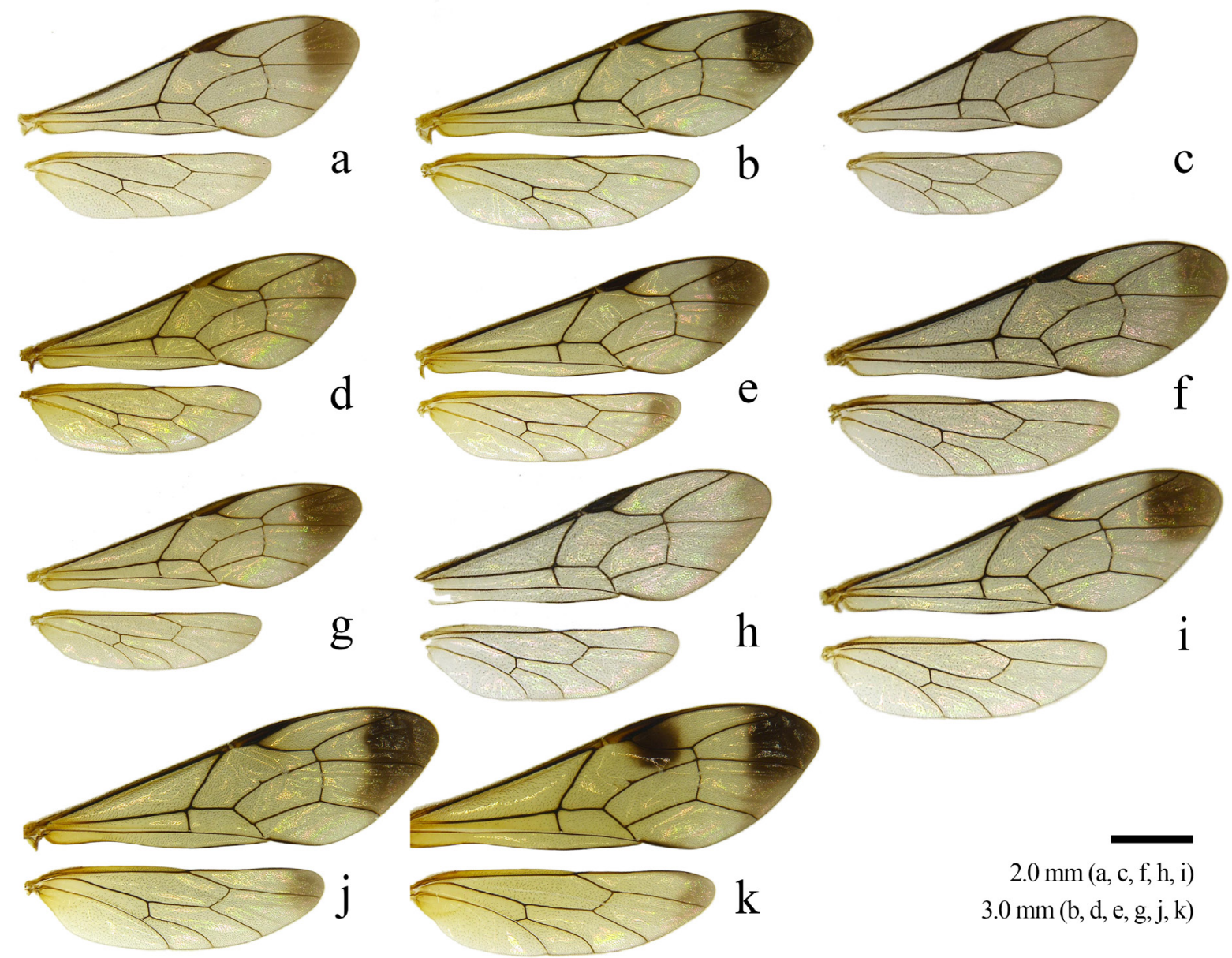

Fig. 4. Wings of females. a. Spilopteron albiventre sp. nov., paratype, Ehime Pref. b. Spilopteron apicale (Matsumura, 1912), Nagano Pref. c. Spilopteron brachyurum sp. nov., paratype, Hokkaido. d. Spilopteron luteum (Uchida, 1930), Amami-oshima Is. e. Spilopteron mucronatus Lee, 2008, Fukuoka Pref. f. Spilopteron nigrum sp. nov., paratype, Hyogo Pref. g. Spilopteron oblongulum sp. nov., paratype, Kanagawa Pref. h. Spilopteron pseudonigrum sp. nov., paratype, Hyogo Pref. i. Spilopteron pyrrhonae Kusigemati, 1981, Kanagawa Pref. j. Spilopteron tosaense (Uchida, 1934), Hokkaido. k. Spilopteron tosaense (Uchida, 1934), Yakushima Is. 
flattened in lateral view (Fig. 5c). Metapleuron sparsely punctate (Fig. 5c). Propodeum strongly carinate (Fig. 6c). Area superomedia rounded. Area externa weakly punctate (Fig. 6c). Area basalis, area superomedia and area dentipara polished (Fig. 6c). Area posteroexterna confluent with area petiolaris and polished (Fig. 6c). Fore wing length $6.5-8.0 \mathrm{~mm}$. Vein $\mathrm{cu}-a$ basad of vein $R s \& M$ (Fig. 4c). Vein $r s-m$ opposite or basad of vein 2m-cu (Fig. 4c). Hind femur 3.1-3.6 times as long as maximum depth in lateral view, without distinct convexity ventrally. Hind tibia 9.0 times as long as maximum depth in lateral view. First hind tarsomere 2.6-2.8 times as long as second and 2.8-3.0 times as long as longer hind tibial spur.

Metasoma. T1 1.9-2.2 times as long as maximum width, 2.3-2.4 times as long as T2. T2 0.5-0.6 times as long as maximum width. T1 weakly and sparsely punctate. T2-T8 weakly and densely punctate. S1 without distinct sharp projection basally. Ovipositor sheath 1.9 times as long as hind tibia.

Color. Body black (Fig. 1c). Antennal flagellum with a white band (Fig. 1c). Inner margin of eye yellowish white (Fig. 2c). Basal area of mandible, face, clypeus, and fore and mid legs brown. Fore wing with a weakly infuscate apical area, extending downwards (Fig. 4c).

Male $(\mathrm{n}=8$; genitalia, $\mathrm{n}=2)$

Similar to female (Fig. 1d). Body length 7.5-9.0 mm. Face 0.6-0.7 times as long as wide. Length of malar space 0.8-0.9 times as long as basal mandibular width. Ocello-ocular line/lateral ocellar diameter = 1.2-1.6. Postocellar line/lateral ocellar diameter $=0.8-1.2$. Antenna with 35-38 flagellomeres; first flagellomere 1.0-1.3 times as long as second flagellomere. Fore wing: length 7.5-9.0 mm. Hind tibia 9.6-10.7 times as long as maximum depth in lateral view. First hind tarsomere 2.0-2.5 times as long as second and 2.5-2.6 times as long as longer hind tibial spur. T1 2.1-2.5 times as long as maximum width, 2.0-2.1 times as long as T2. T2 0.6-0.8 times as long as maximum width. Face, clypeus, basal area of mandible, hind tarsus and apices of $\mathrm{T} 1$ and $\mathrm{T} 2$ yellowish white. Fore and mid legs yellowish white with brown markings. Apex of hind tarsus yellowish white (Fig. 1d).

Subgenital plate pentagonal with obtuse basal angle (Fig. 8c), its length ca 0.7 times as long as aedeagus. Paramere short, basal part strongly projecting towards base of subgenital plate (Fig. 7e). Aedeagus gently curved, its penis valve ca 2.0 times as long as basal apodeme (Fig. 7f).

\section{Distribution}

Japan (Hokkaido and Honshu).

\section{Bionomics}

Host unknown. Adults mainly fly in July.

Spilopteron luteum (Uchida, 1930)

Figs $2 \mathrm{~d}, 3 \mathrm{~d}, 4 \mathrm{~d}, 5 \mathrm{~d}, 6 \mathrm{~d}, 7 \mathrm{~g}-\mathrm{h}, 8 \mathrm{~d}$

Acoenitus luteus Uchida, 1930: 126. Type locality: Taiwan, Urai.

Arotes luteus - Uchida 1932: 141.

Siphimedia lutea - Cushman 1933: 12. — Uchida 1934: 52. — Sonan 1936: 249.

Spilopteron luteum - Townes et al. 1961: 325. - Chiu 1971:22.-Yu et al. 2005, 2012. — Ito et al. 2012: 443.

\section{Material examined}

TAIWAN: 1 (type of Acoenitus luteus), Urai, 20. Jul. 1929, C. Watanabe (SEHU). 
JAPAN: Amami-oshima Is., Kagoshima Pref., 1 q, Kamiya, 1. Aug. 1992, R. Noda (NIAES); 9 q $q$, Mt Yui-dake, 2 Jul. 2004; 2 우, same locality, 15 Jul. 2004, 1 ㅇ, same locality, Malaise trap, 10 Aug. 2004, H. Makihara (NIAES); 1 ${ }^{\lambda}$, same locality, 6 Jul. 2013, M. Ito (KPMNH); 1 d, Wase-toge, 25 Jun. 2010;

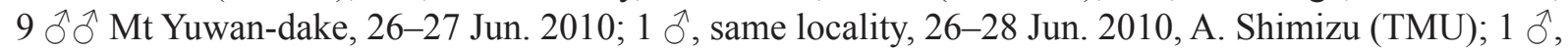
Mt Akatsuchi-yama, 27 Jun. 2011, H. Sawada (LC041303 OMNH); 1 , same locality, 28 Jun. 2011, K. Watanabe (LC041304 OMNH); 2 우, same locality, Malaise trap, 28 Jun.-4 Jul. 2011, K. Kikuchi, T. Yoshida, K. Watanabe and S. Fujie (KPMNH); 1 O, Oodana, Yamato-son, 3 Jul. 2011, H. Sawada (TUA); 1 O, same locality, 4 Jul. 2011, K. Watanabe (NSMT); 1 \&, same locality, 4 Jul. 2011, S. Fujie (NSMT).
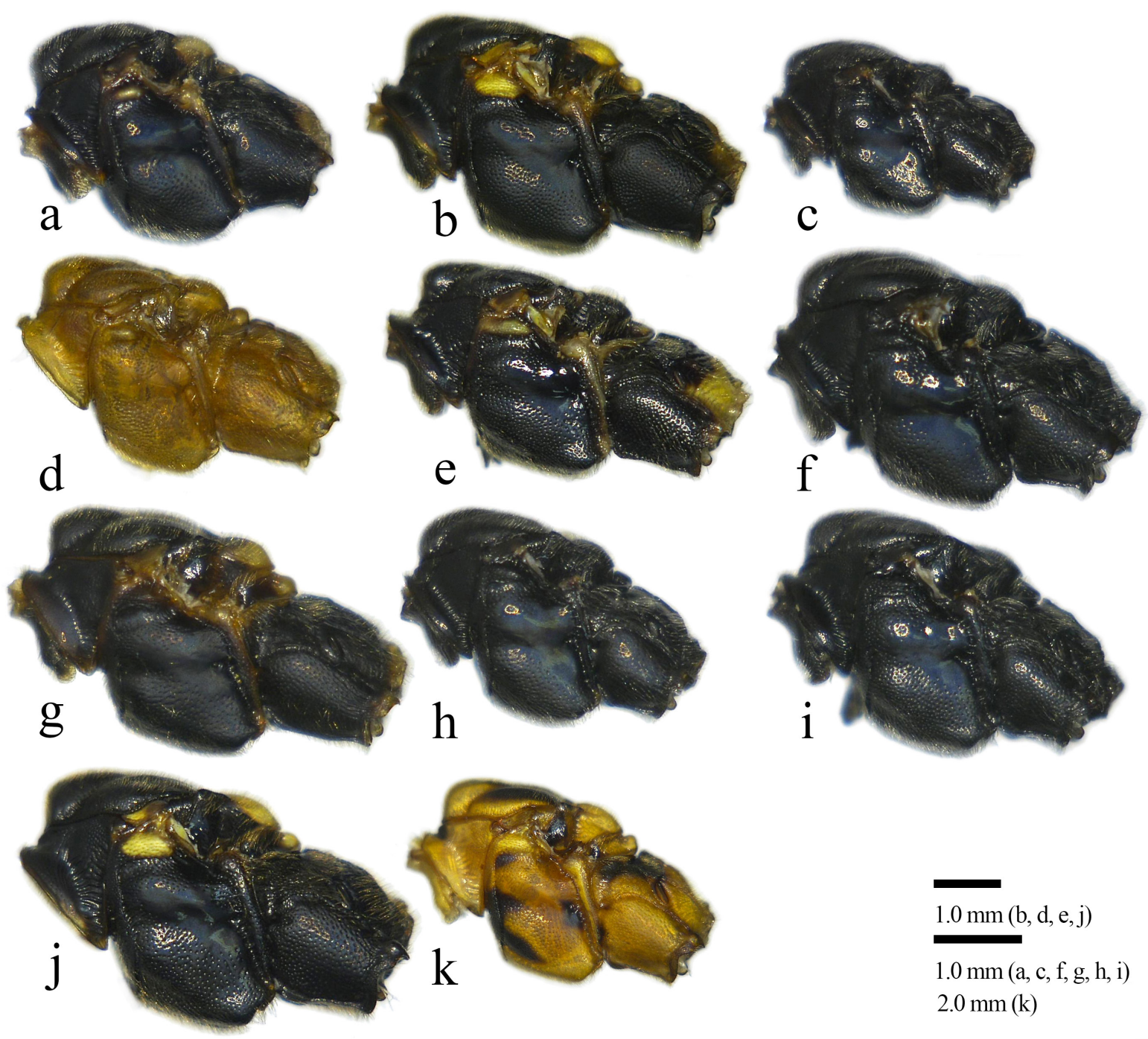

Fig. 5. Mesosoma of females, lateral view. a. Spilopteron albiventre sp. nov., paratype, Ehime Pref. b. Spilopteron apicale (Matsumura, 1912), Nagano Pref. c. Spilopteron brachyurum sp. nov., paratype, Hokkaido. d. Spilopteron luteum (Uchida, 1930), Amami-oshima Is. e. Spilopteron mucronatus Lee, 2008, Fukuoka Pref. f. Spilopteron nigrum sp. nov., paratype, Hyogo Pref. g. Spilopteron oblongulum sp. nov., Kanagawa Pref. h. Spilopteron pseudonigrum sp. nov., paratype, Hyogo Pref. i. Spilopteron pyrrhonae Kusigemati, 1981, Kanagawa Pref. j. Spilopteron tosaense (Uchida, 1934), Hokkaido. k. Spilopteron tosaense (Uchida, 1934), Yakushima Is. 


\section{Differential diagnosis}

This species can easily be distinguished from other Japanese species of Spilopteron by the entirely yellowish brown body (largely black or at least with black areas in other Japanese species) (Ito et al. 2012). ML analysis indicates that this species is most closely related to $S$. tosaense (Fig. 9), and this is also supported by the morphological similarity (except for the body coloration).

\section{Description}

$$
\text { Female }(\mathrm{n}=18)
$$

Body LENGTH. 10.5-14.0 mm.

HEAD. Vertex 0.5-0.6 times as long as maximum length of eye in dorsal view. Frons without a depression between eye and antennal socket in frontal view (Fig. 2d). Clypeus 0.6 times as long as wide, with two strong lateral projections and a weak median projection (Figs 2d, 3d). Face 0.7 times as long as wide. Length of malar space $0.8-1.0$ times as long as basal mandibular width. Ocello-ocular line/lateral ocellar diameter $=2.0-2.1$. Postocellar line/lateral ocellar diameter $=1.0-1.1$. Antenna with 33-38 flagellomeres; first flagellomere 1.2-1.3 times as long as second flagellomere.

Mesosoma. Propleuron densely punctate (Fig. 5d). Lateral area of pronotum densely punctate and transversely striate medially (Fig. 5d). Collar weakly punctate. Mesoscutum densely punctate. Subalar prominence extensively punctate (Fig. 5d). Scutellum weakly punctate in dorsal view. Postscutellum roundly convex in lateral view (Fig. 5d). Metapleuron densely punctate (Fig. 5d). Propodeum strongly carinate (Fig. 6d). Area externa weakly punctate (Fig. 6d). Area basalis polished (Fig. 6d). Area dentipara, area posteroexterna and area petiolaris confluent with area superomedia (Fig. 6d). Fore wing length $10.5-14.0 \mathrm{~mm}$. Vein $c u$ - $a$ basad of vein $R s \& M$ (Fig. 4d). Vein $r s-m$ opposite or distad of vein $2 m-c u$ (Fig. 4d). Hind femur 3.4-3.6 times as long as maximum depth in lateral view, without distinct convexity ventrally. Hind tibia 10.4-12.6 times as long as maximum depth in lateral view. First hind tarsomere 2.3-2.8 times as long as second and 2.4-2.8 times as long as longer hind tibial spur.

Metasoma. T1 2.5-2.9 times as long as maximum width, 2.4-2.6 times as long as T2. T2 0.6-0.8 times as long as maximum width. T1 weakly and sparsely punctate. T2-T8 weakly and densely punctate. S1 without distinct sharp projection basally. Ovipositor sheath 1.7-1.8 times as long as hind tibia.

Color. Body reddish yellow (Fig. 5d). Antennal flagellum brown, usually with a distinct white band, but sometimes band not clear. Apex of mandible black (Fig. 3d). Apical area of hind trochantellus and basal area of hind femur brown. Fore wing with an apical dark mark extending downwards (Fig. 4d).

Male $(\mathrm{n}=15$; genitalia, $\mathrm{n}=2)$

Similar to female. Clypeus 0.6-0.7 times as long as wide. Antenna with 39-42 flagellomeres; first flagellomere 1.1-1.2 times as long as second flagellomere. Hind femur 4.4 times as long as maximum depth in lateral view. Hind tibia 13.4-14.2 times as long as maximum depth in lateral view. First hind tarsomere 2.1 times as long as second and 2.9-3.1 times as long as longer hind tibial spur. T1 3.1-3.2 times as long as maximum width, 2.3-2.4 times as long as T2. T2 0.9 times as long as maximum width. Antennal flagellum black, without a white band.

Subgenital plate hexagonal with basal angle obtuse (Fig. 8d). Paramere short, basal part strongly projecting towards base of subgenital plate (Fig. $7 \mathrm{~g}$ ). Aedeagus gently curved, its penis valve ca 1.6 times as long as basal apodeme (Fig. 7h).

\section{Distribution}

Japan (Amami-oshima Is.), and Taiwan. 


\section{Bionomics}

Host unknown. Adults mainly fly in June and July.

Spilopteron mucronatus Lee, 2008

Figs 2e, 3e, 4e, 5e, 6e, 7i-j, 8e

Spilopteron mucronatus Lee, 2008 in Lee et al. 2008: 276. Type locality: Korea, Gyeonggi-do Province, Yangpyeong-gun Yongnum-myeon Mt Yongmun.

Spilopteron mucronatus - Yu et al. 2012. - Ito et al. 2012: 445.

\section{Material examined}

JAPAN: 1 ð̊, Soun-kyo, Kamikawa-cho, Hokkaido Pref., 13 Jul. 2012, M. Ito (LC041302, NIAES); 1 q, Mt Teinekanayama, Sapporo city, Hokkaido Pref., 19 Jul. 2012, Y. Nakatani (NSMT); 1 , Rishiri Is., Hokkaido, 15 Jul. 1975, E. Nishida (OMNH); 1 , Mt Hayachinesan, Iwate Pref., 25 Jul.-2 Aug. 1989, M. Sharkey and H. Makihara (NIAES); 1 9, Hanase-toge, Sakyo-ku, Kyoto Pref., 23 Jun. 2012, M. Ito (LC041301 NIAES); 1 क, Ideno, Mitsuse-mura, Saga Pref., 17 May 1998, R. Matsumoto (OMNH); 3 우, Mt Hiko-san, Soed-cho, Fukuoka Pref., 15 Jun. 1996, R. Matsumoto (OMNH); 1 , Mt Taterasan, Tsushima Is., Nagasaki Pref., 28 May 1997, N. Takahashi (OMNH); 1 ð , Kamisaka, Tsushima Is., Nagasaki Pref., 3 Jun. 1996, R. Matsumoto (OMNH).

\section{Differential diagnosis}

This species can easily be distinguished from other species of Spilopteron by the ventral convexity of the hind femur, and the distinct sharp projection on S1 (see Ito et al. 2012).

\section{Description}

$$
\text { Female }(\mathrm{n}=8)
$$

Body LENGTH. $11.5-15.0 \mathrm{~mm}$.

HEAD. Vertex 0.7-0.9 times as long as maximum length of eye in dorsal view. Frons without a depression between eye and antennal socket in frontal view (Fig. 2e). Clypeus 0.5-0.6 times as long as wide, with two strong lateral projections and a weak median projection (Figs 2e, 3e). Face 0.6 times as long as wide. Length of malar space $0.9-1.0$ times as long as basal mandibular width. Ocello-ocular line/lateral ocellar diameter $=2.0-2.1$. Postocellar line/lateral ocellar diameter $=1.4-1.6$. Antenna with 33-35 flagellomeres; first flagellomere 1.2-1.4 times as long as second flagellomere.

Mesosoma. Propleuron densely punctate (Fig. 5e). Lateral area of pronotum densely punctate and transversely striate medially (Fig. 5e). Collar densely punctate. Mesoscutum densely punctate. Subalar prominence densely punctate (Fig. 5e). Scutellum strongly and sparsely punctate in dorsal view. Postscutellum flattened in lateral view (Fig. 5e). Metapleuron strongly punctate (Fig. 5e). Propodeum weakly carinate (Fig. 6e). Area externa densely punctate (Fig. 6e). Area basalis polished (Fig. 6e). Area dentipara confluent with area superomedia and transversely carinate (Fig. 6e). Area posteroexterna confluent with area petiolaris and transversely carinate (Fig. 6e). Fore wing length 9.0-12.0 mm. Vein $c u-a$ basad of vein $R s \& M$ (Fig. 4e). Vein $r s-m$ opposite or basad of vein $2 m-c u$ (Fig. 4e). Hind femur 3.1-3.5 times as long as maximum depth in lateral view, with a distinct convexity ventrally. Hind tibia 10.6-12.0 times as long as maximum depth in lateral view. First hind tarsomere 2.5-3.2 times as long as second and 3.0-3.6 times as long as longer hind tibial spur. 
Metasoma. T1 2.7-2.9 times as long as maximum width, 2.3-2.4 times as long as T2. T2 0.7-0.8 times as long as maximum width. T1 and T2 weakly and sparsely punctate. T2-T8 weakly and densely punctate. S1 with a distinct sharp projection basally. Ovipositor sheath 2.7-3.0 times as long as hind tibia.

CoLor. Body black (Fig. 5e). Antennal flagellum without a white band, brown apically. Face and postscutellum yellow with black marking medially, but sometimes entirely black except for inner margin of eye. Clypeus, subalar prominence, propodeum, fore and mid legs, hind trochanter, hind trochantellus, apex of hind femur, and apex of T1 yellow. Fore wing with an apical dark mark extending downwards (Fig. 4e).

Male $(\mathrm{n}=2$; genitalia, $\mathrm{n}=2)$

Similar to female. Body length $10.0-13.0 \mathrm{~mm}$. Face $0.6-0.7$ times as long as wide. Length of malar space $0.8-0.9$ times as long as basal mandibular width. Postocellar line/lateral ocellar diameter $=1.4-$ 1.7. Antenna with 37-42 flagellomeres. Hind femur 3.0-3.1 times as long as maximum depth in lateral

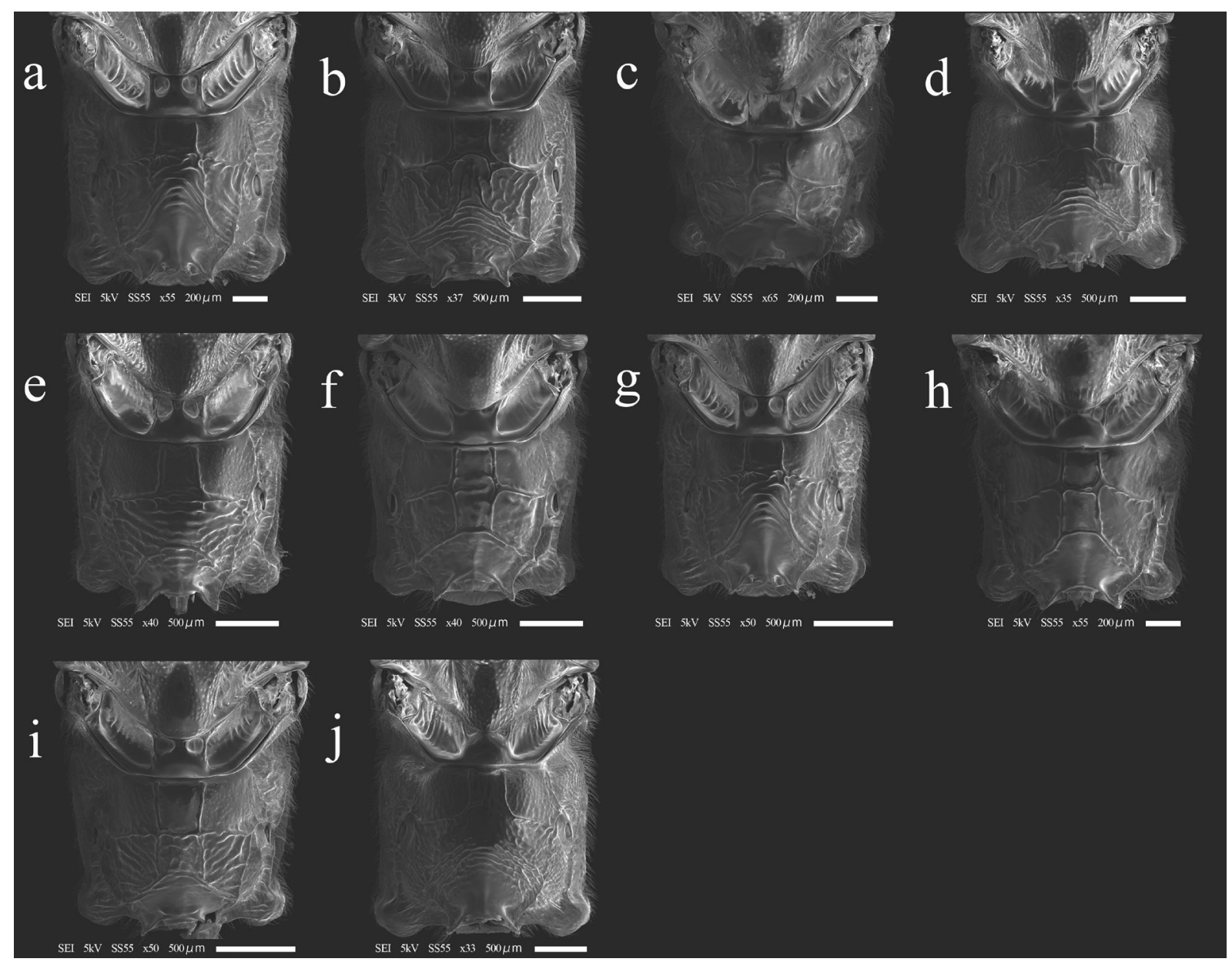

Fig. 6. Propodeum of females, dorsal view. a. Spilopteron albiventre sp. nov., paratype, Ehime Pref. b. Spilopteron apicale (Matsumura, 1912), Nagano Pref. c. Spilopteron brachyurum sp. nov., paratype, Hokkaido. d. Spilopteron luteum (Uchida, 1930), Amami-oshima Is. e. Spilopteron mucronatus Lee, 2008, Fukuoka Pref. f. Spilopteron nigrum sp. nov., paratype, Hyogo Pref. g. Spilopteron oblongulum sp. nov., paratype, Kanagawa Pref. h. Spilopteron pseudonigrum sp. nov., paratype, Hyogo Pref. i. Spilopteron pyrrhonae Kusigemati, 1981, Kanagawa Pref. j. Spilopteron tosaense (Uchida, 1934), Hokkaido. 
view. First hind tarsomere 3.0-3.3 times as long as second. T1 3.1-3.2 times as long as maximum width, 2.0-2.2 times as long as T2. T2 0.8-1.0 times as long as maximum width. Antennal flagellum brown in apical half. Face entirely yellow. Basal area of hind tibia and apex of hind tarsus yellow.

Subgenital plate pentagonal with basal angle obtuse (Fig. 8e). Paramere short, basal part strongly projecting towards base of subgenital plate (Fig. 7i). Aedeagus gently curved, its penis valve ca 2.0 times as long as basal apodeme (Fig. $7 \mathrm{j})$.

\title{
Distribution
}

Japan (Hokkaido, Rishiri Is.*, Honshu, Kyushu, and Tsushima Is.) and Korea. * New record.

\section{Bionomics}

Host unknown. Adults mainly fly from May to July.

\author{
Spilopteron nigrum sp. nov. \\ urn:1sid:zoobank.org:act:B1048E8D-173E-47D0-B0C1-9D38E8802E83
}

Figs 1e-f, 2f, 3f, 4f, 5f, $6 \mathrm{f}$

\section{Etymology}

The specific epithet of this new species is derived from the entirely black body.

\section{Type material}

\section{Holotype}

JAPAN: + , "Mt. Mikuniyama, Katsuragi-cho, Wakayama Pref., Japan, 4 July 2003, R. Matsumoto leg." (OMNH).

\section{Paratypes}

JAPAN: 3 우, Mt Soranumadake, Sapporo-shi, Hokkaido, Malaise trap, 4-27 Jul. 2007, A. Ueda $(\mathrm{OMNH}) ; 2$ 우, same locality, Malaise trap, 27 Jul.-21. Aug. 2007, A. Ueda (KPMNH); 1 , , Ashorocho, Hokkaido, Malaise trap, 15 Jul.-7 Aug. 2008, A. Ueda (OMNH); 1 , Nippu, Bifuka-cho, Hokkaido, Malaise trap, 19 Jun.-17 Jul. 2010, N. Kuhara and M. Sueyoshi (OMNH); 1 \&, Shiretoko, Hokkaido, 13 Aug. 1996, T. Tachi (OMNH); 1 क, Nishirappu, Furano, Hokkaido, 26 Jun.-17 Jul. 2009, A. Ueda (OMNH); 2 우, Okawa, Nishimeya-mura, Aomori Pref., Malaise trap, 21-30 Jun. 2013, T. Nakamura (LC154920, LC154921 EUM); 2 우, Biwakubo-sawa, Masutomi, Hokuto-shi, Yamanashi Pref., 28 Jul. 2007, K. Watanabe (KPMNH); 2 우, same locality, Malaise trap, 28 Jul.-7 Aug. 2007, K. Watanabe (KPMNH); 1 + , Otaki-fudou, Katsunuma-cho, Koushu-shi, Yamanashi Pref., 9 Jul. 2007, K. Watanabe (KPMNH); 2 우, Gozaishi-onsen, Nirasaki-shi, Yamanashi Pref., Malaise trap, 8-17 Jul. 2005, K. Hosoda (OMNH); 5 우, Mt Kariyasu-yama, Kaga-shi, Ishikawa Pref., 28 Jun.-18 Jul. 2002, K. Esaki (OMNH); 1 q, same locality, 28 Jun.-19 Jul. 2002, K. Esaki (SEHU); 3 우, same locality, 19-31 Jul. 2002, K. Esaki (SEHU); 1 q, Hikagedaira, Gifu Pref., 24 Sep. 1980, E. Nishida (OMNH); 1 q, Utsukushimatsu, Nagawa-cho, Nagano Pref., 23 Aug. 2012, S. Fujie (KPMNH); 2 우, Nakajima, Onoshi, Fukui Pref., Malaise trap, 22 Jun.-13 Jul. 2014, S. Fujie and T. Hirooka (LC154917, LC154919 KPMNH); 1 , Akasai-keikoku, Haga-cho, Hyogo Pref., 16 Jun. 2009, R. Matsumoto (OMNH); 2 우, Mt Hyonosen, Yabu-shi, Hyogo Pref., Malaise trap, 16 Jun.-14 Jul. 2013, M. Ito and S. Fujie (NIAES); 3 우, Niiya, Kami-cho, Hyogo Pref., Malaise trap, 16 Jun.-14 Jul. 2013, M. Ito and S. Fujie (NIAES);

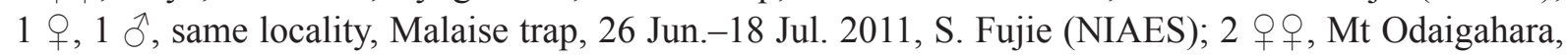
Nara Pref., Malaise trap, 25 Jul.-1 Aug. 2005, A. Kawazoe (EUM). 


\section{Differential diagnosis}

This new species most resembles $S$. pseudonigrum sp. nov., but is easily distinguished from it by the distinct apical marking on the fore wing (the apical portion is only infuscate in S. pseudonigrum sp. nov.).

\section{Description}

$$
\text { Female }(\mathrm{n}=41)
$$

Body LeNGTH. 10.0-12.0 mm.

HEAD. Vertex 0.4-0.5 times as long as maximum length of eye in dorsal view. Frons without a depression between eye and antennal socket in frontal view (Fig. 2f). Clypeus $0.5-0.6$ times as long as wide, with three weak projections of equal size (Fig. 2f). Face 0.6-0.7 times as long as wide. Length of malar space 1.1-1.2 times as long as basal mandibular width. Ocello-ocular line/lateral ocellar diameter $=1.1-1.7$. Postocellar line/lateral ocellar diameter $=1.0-1.3$. Antenna with 28-30 flagellomeres; first flagellomere 1.3-1.4 times as long as second flagellomere.

Mesosoma. Propleuron densely punctate (Fig. 5f). Lateral area of pronotum densely punctate and transversely striate medially. Collar weakly punctate. Mesoscutum densely punctate (Fig. 5f). Subalar prominence extensively punctate (Fig. 5f). Scutellum densely punctate in dorsal view. Postscutellum flattened in lateral view (Fig. 5f). Metapleuron strongly punctate (Fig. 5f). Propodeum strongly carinate (Fig. 6f). Area externa and area dentipara sparsely punctate (Fig. 6f). Area basalis and area superomedia polished (Fig. 6f). Area posteroexterna confluent with area petiolaris and polished (Fig. 6f). Fore wing length $6.0-8.5 \mathrm{~mm}$. Vein $c u$ - $a$ basad of vein $R s \& M$ (Fig. 4f). Vein $r s-m$ opposite or distad of vein $2 m-c u$ (Fig. 4f). Hind femur 3.4-3.6 times as long as maximum depth in lateral view, without distinct convexity ventrally. Hind tibia 9.4-11.3 times as long as maximum depth in lateral view. First hind tarsomere 2.4-2.8 times as long as second and 2.2-2.5 times as long as longer hind tibial spur.

Metasoma. T1 2.0-2.3 times as long as maximum width, 2.4-2.6 times as long as T2. T2 0.6 times as long as maximum width. T1 weakly and sparsely punctate. T2-T8 weakly and densely punctate. S1 without distinct sharp projection basally. Ovipositor sheath 2.1-2.3 times as long as hind tibia.

Color. Body black (Fig. 1e). Antennal flagellum with a white band (Fig. 1e). Inner margin of eye and middle area of mandible yellowish white (Fig. 2f). Fore and mid legs grayish brown (Fig. 1e). Apex of hind tarsus sometimes yellowish white (Fig. 1e). Fore wing with an apical dark mark, extending downwards (Fig. 4f).

$$
\text { Male }(n=1) \text {. }
$$

Similar to female (Fig. 1f). Body length: $9.0 \mathrm{~mm}$. Head 0.7 times as long as wide in dorsal view. Face 0.8 times as long as wide. Antenna with 36 flagellomeres; first flagellomere 1.1 times as long as second flagellomere. Fore wing length $8.0 \mathrm{~mm}$. Hind femur 3.2 times as long as maximum depth in lateral view. First hind tarsomere 3.7 times as long as longer hind tibial spur. T1 2.5 times as long as maximum width, 2.3 times as long as T2. T2 1.0 times as long as maximum width. Antennal flagellum without a white band (Fig. 1f). Face, clypeus, basal area of mandible, apices of T1-3, and fore and middle legs yellowish white.

Male genitalia of this species were not examined due to the limited number of specimens.

\section{Distribution}

Japan (Hokkaido and Honshu). 

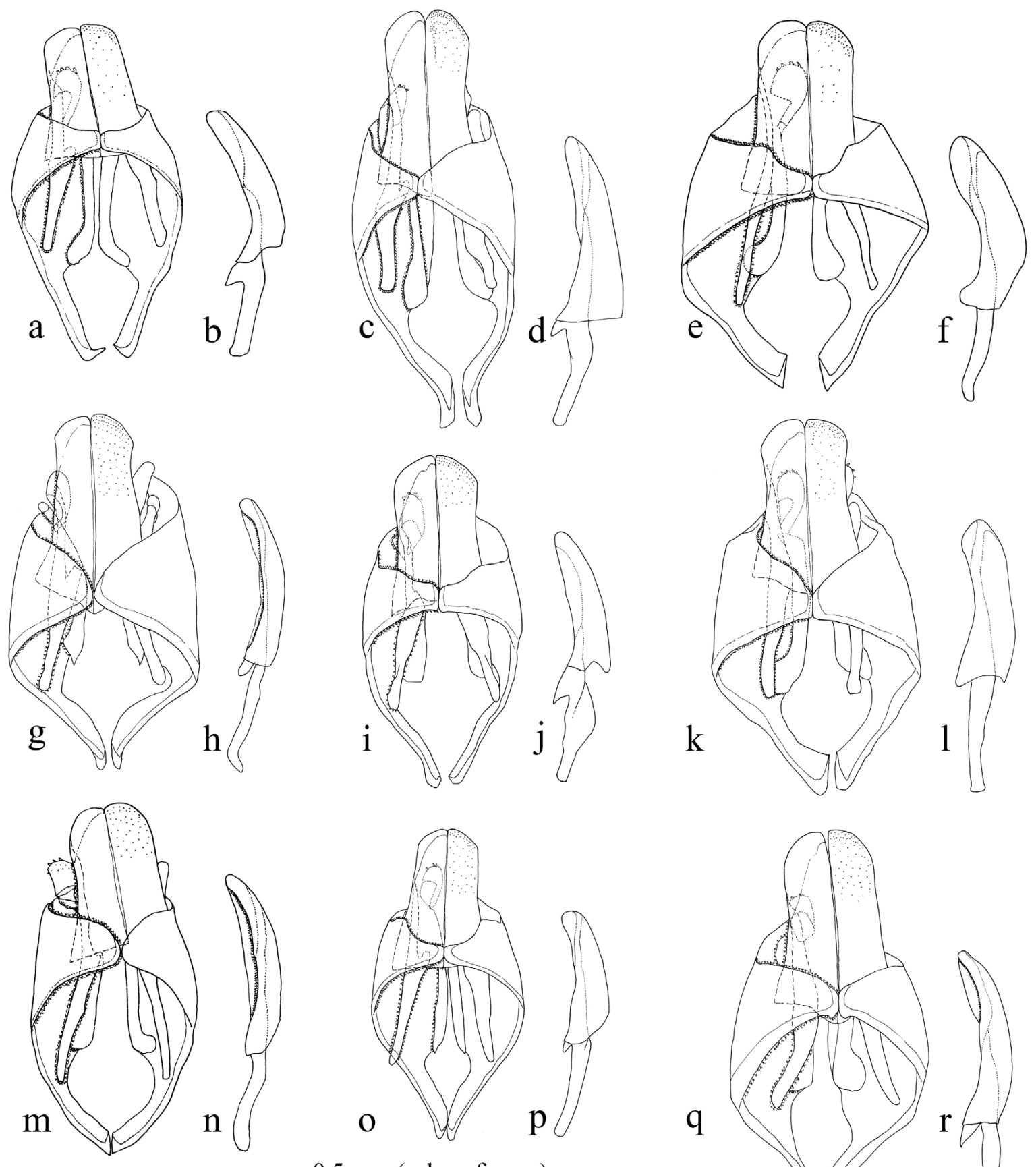

$0.5 \mathrm{~mm}(\mathrm{a}, \mathrm{b}, \mathrm{e}, \mathrm{f}, \mathrm{m}, \mathrm{n})$

$0.5 \mathrm{~mm}(\mathrm{c}, \mathrm{d}, \mathrm{g}, \mathrm{h}, \mathrm{i}, \mathrm{j}, \mathrm{k}, \mathrm{l}, \mathrm{o}, \mathrm{p}, \mathrm{q}, \mathrm{r})$

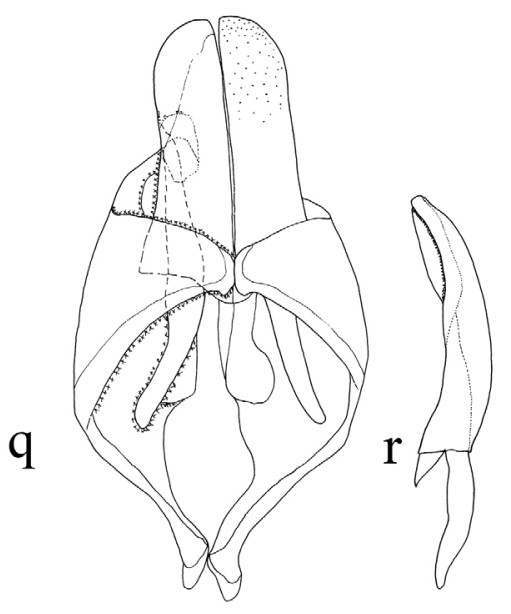

Fig. 7. Male genitalia. a-b. Spilopteron albiventre sp. nov., paratype, Ehime Pref. c-d. Spilopteron apicale (Matsumura, 1912), Nagano Pref. e-f. Spilopteron brachyurum sp. nov., paratype, Hokkaido. g-h. Spilopteron luteum (Uchida, 1930), Amami-oshima Is. i-j. Spilopteron mucronatus Lee, 2008, Hokkaido. k-l. Spilopteron oblongulum sp. nov., paratype, Gunma Pref. m-n. Spilopteron pseudonigrum sp. nov., paratype, Hyogo Pref. o-p. Spilopteron pyrrhonae Kusigemati, 1981, Shizuoka Pref. q-r. Spilopteron tosaense (Uchida, 1934), Hyogo Pref. a, c, e, g, i, k, m, o, q = genital capsule, ventral view; b, d, f, h, j, $1, \mathrm{n}, \mathrm{p}, \mathrm{r}=$ aedeagus, lateral view. 


\section{Bionomics}

Host unknown. Adults mainly fly in July and August.

Spilopteron oblongulum sp. nov. urn:1sid:zoobank.org:act:E842665F-8874-4BCA-92B0-919E768DADCE

Figs $1 \mathrm{~g}-\mathrm{h}, 2 \mathrm{~g}, 3 \mathrm{~g}, 4 \mathrm{~g}, 5 \mathrm{~g}, 6 \mathrm{~g}, 7 \mathrm{k}-1,8 \mathrm{f}$

\section{Etymology}

The specific epithet of this new species is derived from the long ovipositor sheath.

\section{Type material}

\section{Holotype}

JAPAN: 1 q, “Oku-nikko, Nikko-shi, Tochigi Pref., Japan, 9. August 1980, K. Konishi leg.” (NIAES).

\section{Paratypes}

JAPAN: 1 , Doudaira, Tanzawa, Kanagawa Pref., 29 Aug. 1993 T. Kinoshita (NIAES); 1 q, 1 ภ, Mt Mikuniyama, Yamakita-cho, Kanagawa Pref., 11 Aug. 2015, T. Taniwaki (LC154926, LC154927

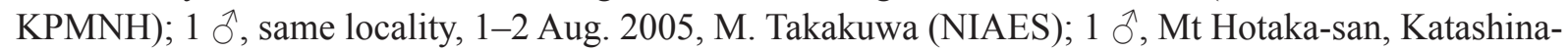
mura, Gumma Pref., 2 Aug. 2007, K. Watanabe (KPMNH); 1 ô, Mt Ontake-san, Outaki-mura, Nagano Pref., 7 Aug. 2010, K. Watanabe (KPMNH).

\section{Differential diagnosis}

This new species most resembles S. flavescutatum Wang, 2004 and S. longitubus Wang, 2004 from China, but is easily distinguished from the former by the long T1, which is 3.5-4.3 times as long as wide (2.7 times in S. flavescutatum), and from the latter by the polished area petiolaris (rugose in S. longitubus); the female antenna has 35 flagellomeres (43 flagellomeres in $S$. longitubus), and the male S1 ends in front of the spiracles of T1 (a little behind in S. longitubus). ML analysis indicates that this species is most closely related to $S$. apicale. Morphologically, this species shares some unique character states with $S$. apicale, such as the shape of clypeus and the depression between the eye and antennal socket, but it has an obviously longer ovipositor.

\section{Description}

Female $(\mathrm{n}=3)$

BODY LENGTH. 13.0-17.0 mm.

HEAD. Vertex 0.5 times as long as maximum length of eye in dorsal view. Frons without a depression between eye and antennal socket in frontal view (Fig. 2g). Clypeus 0.6 times as long as wide, with two lateral projections (Figs $2 \mathrm{~g}, 3 \mathrm{~g}$ ). Face $0.7-0.8$ times as long as wide. Length of malar space 1.0-1.1 times as long as basal mandibular width. Ocello-ocular line/lateral ocellar diameter $=1.4-1.6$. Postocellar line/ lateral ocellar diameter $=1$.1. Antenna with 35 flagellomeres; first flagellomere $1.2-1.3$ times as long as second flagellomere.

Mesosoma. Propleuron densely punctate (Fig. 5g). Lateral area of pronotum densely punctate and transversely striate medially (Fig. 5g). Collar weakly punctate. Mesoscutum densely punctate. Subalar prominence extensively punctate (Fig. 5g). Scutellum densely punctate in dorsal view. Postscutellum roundly convex in lateral view (Fig. 5g). Metapleuron extensively punctate (Fig. 5g). Propodeum strongly carinate (Fig. 6g). Area externa densely punctate (Fig. 6g). Area basalis, area dentipara and area superomedia weakly carinate (Fig. 6g). Area posteroexterna confluent with area petiolaris (Fig. 6g). Fore wing length $11.5-14.0 \mathrm{~mm}$. Vein $c u-a$ basad of vein $R s \& M$ (Fig. 4g). Vein $r s-m$ opposite or basad 
of vein $2 m-c u$ (Fig. 4g). Hind femur 4.7-5.1 times as long as maximum depth in lateral view, without distinct convexity ventrally. Hind tibia 13.3-13.5 times as long as maximum depth in lateral view. First hind tarsomere 2.3-2.6 times as long as second and 3.5-3.8 times as long as longer hind tibial spur.

Metasoma. T1 2.9-3.7 times as long as maximum width, 2.1-2.2 times as long as T2. T2 0.9-1.0 times as long as maximum width. T1 weakly and sparsely punctate. T2-T8 weakly and densely punctate. S1 without distinct sharp projection basally. Ovipositor sheath 2.3-2.6 times as long as hind tibia.

Color. Body black (Fig. 5g). Antennal flagellum with a white band (Fig. 1g). Inner margin of eye, clypeus, basal area of mandible, scutellum, postscutellum, area petiolaris and apices of T1 and T2 yellow. Fore and mid legs, trochanter, trochantellus and hind tarsus tinged yellowish brown. Fore wing with an apical dark mark extending downwards (Fig. 4g).

Male $(\mathrm{n}=4$; genitalia, $\mathrm{n}=2)$

Similar to female (Fig. 1h). Vertex $0.6-0.7$ times as long as maximum length of eye in dorsal view. Face $0.7-0.8$ times as long as wide. Ocello-ocular line/lateral ocellar diameter $=1.3-1.5$. Postocellar line/ lateral ocellar diameter $=0.9-1.3$. Antenna with 39-41 flagellomeres; first flagellomere 1.0-1.2 times as long as second flagellomere. Fore wing length 12.0-14.5 mm. Hind femur 5.5-5.7 times as long as maximum depth in lateral view. Hind tibia 12.6-14.0 times as long as maximum depth in lateral view. First hind tarsomere 2.2-2.4 times as long as second and 4.3-4.8 times as long as longer hind tibial spur. T1 3.5-4.3 times as long as maximum width. S1 in front of spiracles of T1. T2 1.0-1.1 times as long as maximum width. Antennal flagellum yellowish brown, without a white band (Fig. 1h). Face, basal area of $\mathrm{T} 1$ yellowish brown. Hind femur and tibia yellow to brown.

Subgenital plate hexagonal with basal angle obtuse (Fig. 8f). Paramere short, basal part strongly projecting towards base of subgenital plate (Fig. $7 \mathrm{k}$ ). Aedeagus gently curved, its penis valve ca 2.0 times as long as basal apodeme (Fig. 71).

\section{Distribution}

Japan (Honshu).

\section{Bionomics}

Host unknown. Adults fly in August.

Spilopteron pseudonigrum sp. nov. urn:1sid:zoobank.org:act:649F2A74-91F4-44EB-A17B-0F9687FE819D

Figs $1 \mathrm{i}-\mathrm{j}, 2 \mathrm{~h}, 3 \mathrm{~h}, 4 \mathrm{~h}, 5 \mathrm{~h}, 6 \mathrm{~h}, 7 \mathrm{~m}-\mathrm{n}, 8 \mathrm{~g}$

\section{Etymology}

The specific epithet of this new species is derived from the similarity to $S$. nigrum sp. nov.

\section{Type material}

Holotype

JAPAN: +, "Mt. Hyonosen, Yabu-shi, Hyogo Pref., Japan, 16 June-14 July 2013, M. Ito \& S. Fujie leg." Malaise trap, $(\mathrm{OMNH})$.

\section{Paratypes}

JAPAN: $7 \hat{\partial}$, Kawaratai, Nishimeya-mura, Aomori Pref., Malaise trap, 12-21 Jun. 2013, T. Nakamura (LC154923, LC154924 EUM); 6 $\widehat{\partial} \widehat{\partial}$, same locality, Malaise trap, 21-30 Jun. 2013, T. Nakamura 
(EUM); 2 우, $5 \widehat{\delta} \widehat{\partial}$, same locality, Malaise trap, 30 Jun.-15 Jul. 2013, T. Nakamura (LC154922 EUM); 1 , Asahi-kosen, Yamagata Pref., 23 Jul. 1971, A. Nagatomi (SEHU), 1 ô, Mt Yudono-san, Asahi-mura, Yamagata Pref., 12 Aug. 1996, N. Takahashi (OMNH); 1 q, Hinoemata-mura, Fukushima Pref., 24-25 Aug. 1989, K. Konishi (NIAES); 1 q, Suginosawa, Myoko-shi, Niigata Pref., 3 Aug. 2012, M. Ito (KPMNH); 1 \&, same locality, 16 Jul. 2013, S. Shimizu (KPMNH); 1 , same locality, 17 Aug. 2013, S. Shimizu (KPMNH); 2 우, Mt Hotaka-san, Katashina-mura, Gunma Pref., 1 Aug. 2007, T. Ishizaki (KPMNH); 1 q, same locality, 2 Aug. 2007, M. Irie (KPMNH); 1 , , Hirogawara, Ashiyasumura, Yamanashi Pref., 14 Jul. 1996, T. Tachi (OMNH); 1 ㅇ, Mt Hakkaisan, Outaki-mura, Nagano Pref., 28 Jul. 2013, S. Shimizu (KPMNH); 1 क , Hirugano-kogen, Gujo-shi, Gifu Pref., 8 Jul. 2006, H. Yoshida (OMNH); 1 + , Nakajima, Ono-shi, Fukui Pref., Malaise trap, 22 Jun.-13 Jul. 2014, S. Fujie and T. Hirooka (OMNH); 1 ㅇ, Mt Haku-san, Ishikawa Pref., 3 Aug. 1988, I. Togashi (NIAES); 9 q $q$, $1 \lesssim$, same data as holotype (OMNH); 1 q, Mt Koya-yama, Odamiyama, Ehime Pref., 11 Jul. 1994, E. Yamamoto (NIAES).

\section{Differential diagnosis}

This new species most resembles $S$. nigrum sp. nov., although it can be distinguished by the merely infuscate apical area of the fore wing (with a distinct apical mark in S. nigrum sp. nov.). Although S. pseudonigrum sp. nov. and $S$. nigrum sp. nov. are difficult to distinguish from each other, they are distinct by molecular analysis (Fig. 9); the genetic distance between them was about $9.8 \%$. This species also resembles $S$. brachyurum sp. nov., but it has a square area superomedia of the propodeum (the area is rounded in $S$. brachyurum sp. nov.).

\section{Description}

$$
\text { Female }(\mathrm{n}=27) \text {. }
$$

Body LENGTH. 6.5-9.5 mm.

HEAD. Vertex 0.4 times as long as maximum length of eye in dorsal view. Frons without a depression between eye and antennal socket in frontal view (Fig. 2h). Clypeus 0.3-0.6 times as long as wide, with three weak projections of equal size (Figs $2 \mathrm{~h}, 3 \mathrm{~h}$ ). Face 0.7 times as long as wide. Length of malar space 1.1-1.3 times as long as basal mandibular width. Ocello-ocular line/lateral ocellar diameter $=1.3-1.7$. Postocellar line/lateral ocellar diameter $=1.2$. Antenna with 27-28 flagellomeres; first flagellomere 1.21.4 times as long as second flagellomere.

Mesosoma. Propleuron densely punctate (Fig. 5h). Lateral area of pronotum densely punctate and transversely striate medially. Collar weakly and sparsely punctate. Mesoscutum densely punctate (Fig. 5h). Subalar prominence extensively punctate (Fig. 5h). Scutellum sparsely punctate in dorsal view. Postscutellum flattened in lateral view (Fig. 5h). Metapleuron sparsely punctate (Fig. 5h). Propodeum strongly carinate (Fig. 6h). Area superomedia square. Area externa and area dentipara sparsely punctate (Fig. 6h). Area basalis and area superomedia polished (Fig. 6h). Area posteroexterna confluent with area petiolaris and polished (Fig. 6h). Fore wing length $6.0-8.5 \mathrm{~mm}$. Vein $c u-a$ basad of vein $R s \& M$ (Fig. $4 \mathrm{~h}$ ). Vein $r s-m$ opposite or distad of vein $2 m-c u$ (Fig. 4 h). Hind femur 3.5-3.7 times as long as maximum depth in lateral view, without distinct convexity ventrally. Hind tibia 8.7-9.5 times as long as maximum depth in lateral view. First hind tarsomere 2.4-2.8 times as long as second one and 2.1-2.4 times as long as longer hind tibial spur.

Metasoma. T1 1.9-2.4 times as long as maximum width, 2.2-2.7 times as long as T2. T2 0.5-0.7 times as long as maximum width. T1 weakly and sparsely punctate. T2-T8 weakly and densely punctate. S1 without distinct sharp projection basally. Ovipositor sheath 2.0-2.3 times as long as hind tibia. 
CoLor. Body black (Fig. 1i). Antennal flagellum with a white band (Fig. 1i). Inner margin of eye and middle area of mandible yellowish white (Fig. 2h). Fore and mid legs grayish brown (Fig. 1i). Apex of hind tarsus sometimes yellowish white (Fig. 1i-j). Fore wing lacking dark marks, with only an infuscate apical area (Fig. 4h).

Male ( $\mathrm{n}=19$; genitalia, $\mathrm{n}=2)$

Similar to female (Fig. 1j). Vertex $0.4-0.5$ times as long as maximum length of eye in dorsal view. Face $0.7-0.8$ times as long as wide. Length of malar space $0.9-1.2$ times as long as basal mandibular width. Ocello-ocular line/lateral ocellar diameter $=1.2-1.4$. Postocellar line/lateral ocellar diameter $=1.0-1.4$. Antenna with 33-36 flagellomeres; first flagellomere 1.1-1.4 times as long as second flagellomere. Hind tibia 8.3-9.6 times as long as maximum depth in lateral view. First hind tarsomere 2.4-2.9 times as long as second and 2.2-2.6 times as long as longer hind tibial spur. T1 2.3-3.2 times as long as maximum width. T2 0.7-0.9 times as long as maximum width. Antennal flagellum without a white band. Face, clypeus, basal area of mandible, apices of T1 and T2, and fore and mid legs yellowish white.
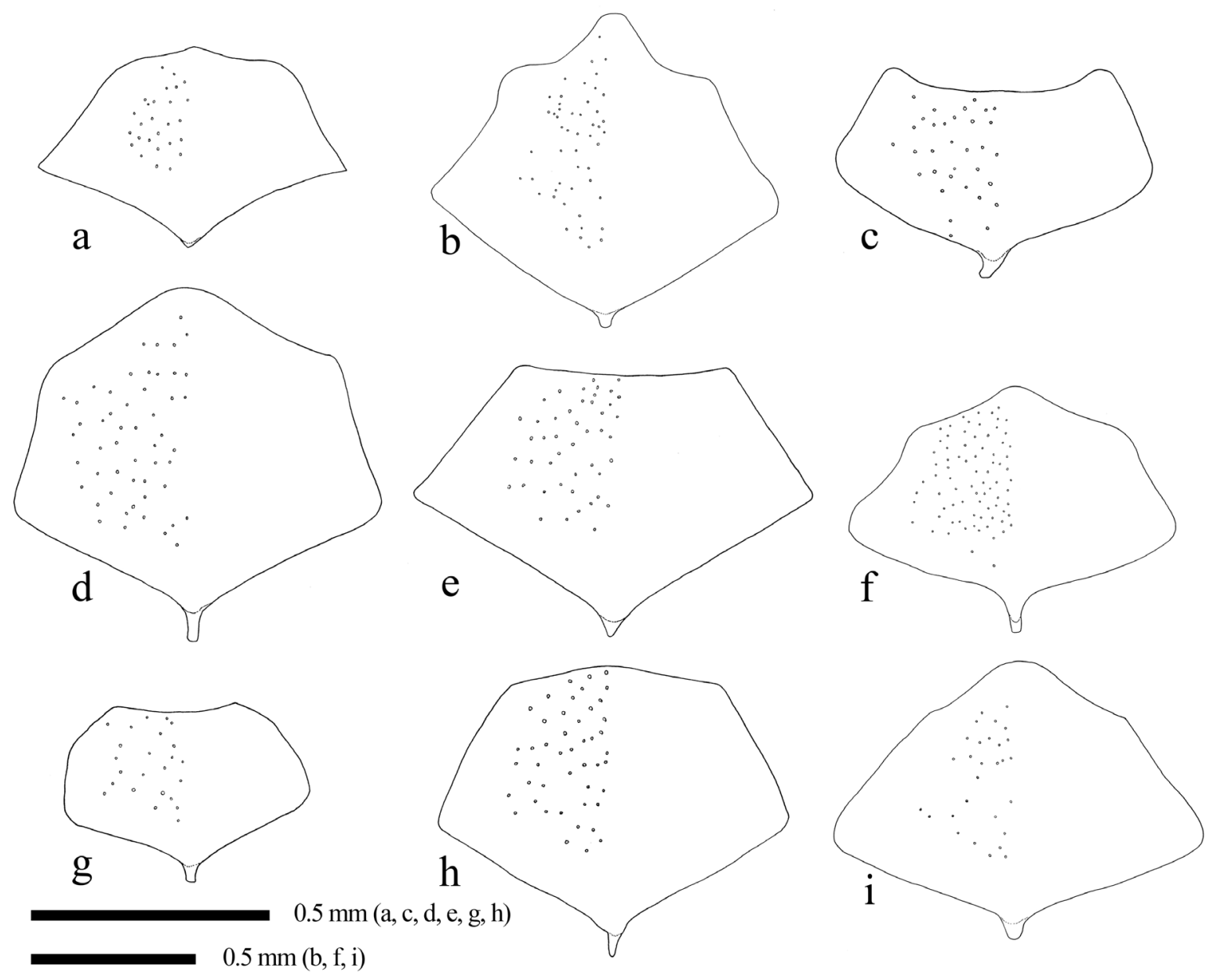

Fig. 8. Male subgenital plate, ventral view. a. Spilopteron albiventre sp. nov., paratype, Ehime Pref. b. Spilopteron apicale (Matsumura, 1912), Yamanashi Pref. c. Spilopteron brachyurum sp. nov., paratype, Hokkaido. d. Spilopteron luteum (Uchida, 1930), Amami-oshima Is. e. Spilopteron mucronatus Lee, 2008, Hokkaido. f. Spilopteron oblongulum sp. nov., paratype, Gunma Pref. g. Spilopteron pseudonigrum sp. nov., paratype, Hyogo Pref. h. Spilopteron pyrrhonae Kusigemati, 1981, Shizuoka Pref. i. Spilopteron tosaense (Uchida, 1934), Hyogo Pref. 
Subgenital plate pentagonal with basal angle obtuse (Fig. 8g). Paramere short, basal part strongly projecting towards base of subgenital plate (Fig. $7 \mathrm{~m}$ ). Aedeagus gently curved, its penis valve ca 2.0 times as long as basal apodeme (Fig. 7n).

\section{Distribution}

Japan (Honshu and Shikoku).

\section{Bionomics}

Host unknown. Adults mainly fly in July and August.

Spilopteron pyrrhonae Kusigemati, 1981

Figs $2 \mathrm{i}, 3 \mathrm{i}, 4 \mathrm{i}, 5 \mathrm{i}, 6 \mathrm{i}, 7 \mathrm{o}-\mathrm{p}, 8 \mathrm{~h}$

Spilopteron pyrrhonae Kusigemati, 1981: 123. Type locality: Japan, Kagoshima Pref.

Spilopteron pyrrhonae - Yu et al. 2005, 2012.

\section{Material examined}

Holotype

JAPAN: 1 \%, Takachiho-gawara, Kagoshima Pref., 24 Apr. 1975, Y. Minami (SEHU).

\section{Other material examined}

JAPAN: 3 qq $q, 2$ $\widehat{\jmath}$, Kamiyu, Hakone-machi, Kanagawa Pref., 20 Dec. 2003 (emerged), K. Watanabe (KPMNH); 1 + 1 §’, Amagi-kogen, Izu-shi, Shizuoka Pref., Apr. 2008 (emerged), H. Kawai (KPMNH); 1 , 1 Ĵ, Amagi-toge, Kawazu-shi, Shizuoka Pref., 27 Apr. 2013 (emerged), A. Kosaki (LC041988, LC041989 OMNH); 1 \&, same locality, 27 Apr. 2013 (emerged), Y. Shiozawa (LC041990 NIAES); 1 , Kurodake, Oita Pref., 8-9 Jun. 1985, T. Hirowatari (NIAES); 1 đ̊, Katamukiyama, Ogata-machi, Oita Pref., 6 Jun. 1992, R. Noda (NIAES); 1 Ĵ, Hikosan, Fukuoka Pref., 19 Apr. 1980 (emerged), R. Noda (NIAES); 1 \&, 1 ô, same locality, Jun. 1982 (emerged), R. Noda (NIAES); 1 \&, same locality, 30 Mar. 1982 (emerged), R. Noda (NIAES).

\section{Differential diagnosis}

This species resembles $S$. brachyurum sp. nov. and S. nigrum sp. nov.; however, it can be distinguished from them by the rounded apical dark mark on the fore wing (extending downwards in S. brachyurum sp. nov. and S. nigrum sp. nov.), and turned up clypeus in lateral view (not turned up in S. brachyurum sp. nov. and $S$. nigrum sp. nov.). It is also easily distinguished from other Japanese Spilopteron by the above character states.

\section{Description}

Female $(\mathrm{n}=11)$

Body LENGTH. 10.5-13.0 mm.

HEAD. Vertex 0.6-0.7 times as long as maximum length of eye in dorsal view. Frons without a depression between eye and antennal socket in frontal view (Fig. 2i). Clypeus 0.6 times as long as wide, with two strong lateral projections, and anterior margin turned up (Figs 2i, 3i). Face 0.6-0.7 times as long as wide. Length of malar space $0.9-1.2$ times as long as basal mandibular width. Ocello-ocular line/lateral ocellar diameter $=1.4-1.8$. Postocellar line/lateral ocellar diameter $=0.9-1.4$. Antenna with 32-33 flagellomeres; first flagellomere 1.3 times as long as second flagellomere. 
Mesosoma. Propleuron densely punctate (Fig. 5i). Lateral area of pronotum densely punctate and transversely striate medially (Fig. 5i). Collar weakly punctate. Mesoscutum densely punctate. Subalar prominence extensively punctate (Fig. 5i). Scutellum weakly punctate in dorsal view. Postscutellum flattened in lateral view (Fig. 5i). Metapleuron weakly punctate (Fig. 5i). Propodeum strongly carinate (Fig. 6i). Area externa and area dentipara punctate (Fig. 6i). Area basalis polished (Fig. 6i). Area superomedia weakly carinate (Fig. 6i). Area petiolaris polished (Fig. 6i). Fore wing length 9.5-10.0 mm. Vein $c u$ - $a$ basad of vein Rs\&M (Fig. 4i). Vein $r s-m$ opposite vein $2 m-c u$ (Fig. 4i). Hind femur 3.5-3.9 times as long as maximum depth in lateral view, without distinct convexity ventrally. Hind tibia $10.8-$ 11.8 times as long as maximum depth in lateral view. First hind tarsomere 2.4-2.6 times as long as second and 2.2-2.5 times as long as longer hind tibial spur.

Metasoma. T1 2.2-2.5 times as long as maximum width, 2.0-2.3 times as long as T2. T2 0.6-0.7 times as long as maximum width. T1 weakly and sparsely punctate. T2-T8 weakly densely punctate. S1 without distinct sharp projection basally. Ovipositor sheath 2.0-2.1 times as long as hind tibia.

Color. Body black (Fig. 5i). Antennal flagellum with a white band. Inner margin of eye and hind tarsomeres 3-5 white to yellowish white. Fore and mid legs brown. Fore wing with a rounded apical dark mark (Fig. 4i).

Male $(\mathrm{n}=7$; genitalia, $\mathrm{n}=2)$

Similar to female. Clypeus $0.6-0.8$ times as long as wide. Length of malar space 1.0-1.3 times as long as basal mandibular width. Ocello-ocular line/lateral ocellar diameter $=1.4-2.0$. Postocellar line/lateral ocellar diameter $=1.1-1.5$. Antenna with 37-39 flagellomeres; first flagellomere 1.2-1.3 times as long as second flagellomere. Hind femur 3.4-4.1 times as long as maximum depth in lateral view. First hind tarsomere 2.1-2.7 times as long as second and 2.7-3.3 times as long as longer hind tibial spur. Metasoma. T1 2.6-3.0 times as long as maximum width, 1.9-2.3 times as long as T2. T2 0.8-1.0 times as long as maximum width. Face, basal area of mandible, hind tarsus and apices of T1 and T2 yellowish white. Fore and mid legs, trochanters and trochantelli yellowish brown.

Subgenital plate pentagonal with basal angle obtuse (Fig. 8h), its length ca 0.7 times as long as aedeagus. Paramere short, basal part strongly projecting towards base of subgenital plate (Fig. 7o). Aedeagus gently curved, its penis valve ca 1.3 times as long as basal apodeme (Fig. 8h).

\section{Distribution}

Japan (Honshu, Shikoku and Kyushu).

\section{Bionomics}

Kusigemati (1981) reported that this species is a parasitoid of Pyrrhona laeticolor (Cerambycidae). Adults fly in May and June.

Spilopteron tosaense (Uchida, 1934)

Figs $2 \mathrm{j}-\mathrm{k}, 3 \mathrm{j}, 4 \mathrm{j}-\mathrm{k}, 5 \mathrm{j}-\mathrm{k}, 6 \mathrm{j}, 7 \mathrm{q}-\mathrm{r}, 8 \mathrm{i}$

Siphimedia apicalis f. tosaensis Uchida, 1934: 53. Type locality: Japan, Kochi Pref.

Siphimedia apicalis var. yakushimemsis Uchida, 1934: 53. Synonymized by Townes et al. (1965).

Spilopteron tosensis - Townes et al. 1965: 39. — Kusigemati 1981: 121.

Spilopteron apicalis - Kusigemati 1981: 118 (in part).

Spilopteron tosaense - Yu et al. 2005, 2012. — Ito et al. 2015: p. 547. 


\section{Material examined}

JAPAN: 1 (holotype of Siphimedia apicalis f. tosaensis), Mt Koeda, Kochi Pref., 12 Jul. 1933, Y. Sugihara (SEHU); 1 क (holotype of Siphimedia apicalis f. yakushimensis), Hananoegawa, Kuriu, Yakushima Is., Kagoshima Pref., 31 Jul. 1929, H. Hori (SEHU); 1 + , Hitsujigaoka, Sapporo-shi, Hokkaido, 26 Jul.-2 Aug. 2008, K. Konishi (EUM); 2 우, same locality, 4-11 Aug., 2008, K. Konishi (EUM); 2 우, same locality, 20-27 Jul. 2010, K. Konishi (EUM); 3 우, same locality, 25 Jul.-1 Aug. 2011, K. Konishi (EUM); 2 우, same locality, 1-8 Aug. 2011, K. Konishi (EUM); 5 우, same locality, 25 Jul.-1 Aug. 2012, K. Konishi (LC041271 EUM); 3 우, same locality, 1-8 Aug. 2012, K. Konishi (EUM); 3 우, Oyubari, Yubari-shi, Hokkaido, 24 Jul.-24 Aug. 2006, A. Ueda (OMNH); 1 , same locality, 3-17 Aug. 2007, A. Ueda (OMNH); 1 \&, Ashoro-cho, Hokkaido, 17 Jul.-7 Aug. 2008, A. Ueda (OMNH); 1 , , Takinoshita, Kuriyama-cho, Hokkaido, 16 Jul.-5 Aug. 2009, A. Ueda (OMNH); 1 q, Mt Hakkodasan, Aomori Pref., 15 Aug. 1927, T. Uchida (SEHU); 1 \&, Kodomari Peninsula, Nakadomaricho, Aomori Pref., 28 Jul. 2013, D. Kato (KPMNH); 1 +, Okawa, Nishimeya-mura, Aomori Pref., 25 Jul.-6 Aug. 2013, T. Nakamura (EUM); 2 우, Kawaratai, Nishimeya-mura, Aomori Pref., 4-19 Aug. 2011, T. Nakamura (EUM); 1 q, Mt Hakaseyama, Showa-mura, Fukushima Pref., 29 Jun.-26 Jul. 1998, T. Muroi and S. Morishita (MU); 1 +, Mt Nokogiriyama, Suyoshi-cho, Nagaoka-shi, Niigata Pref., 21 Jul.-21 Aug. 2014, So Shimizu and R. Shimizu (KPMNH); 1 +, Mt Houhyousan, Oda, Tsukuba-shi, Ibaraki Pref., 28 Jun.-2 Aug. 2013, S. Shimizu (NSMT); 1 ô, Mt Togarisengen, Tojoji, Tsuchiura-shi, Ibaraki Pref., 1 Jul.-5 Aug. 2014, S. Shimizu (NSMT); 1 ㅇ, Kurabuchi-mura, Takasaki-shi, Gunma Pref., 2 Aug. 2002, H. Suzuki (KPMNH); 1 Jै, Ayuta, Mogi-machi, Tochigi Pref., 23 Jul. 2011, M. Inaizumi (KPMNH); 1 ô, Otaki-mura, Chichibu-shi, Saitama Pref., 13-14 Jul. 2002, T. Nambu (KPMNH); 1 ô, Irikawa forest road, Otaki-mura, Chichibu-shi, Saitama Pref., 20-21 Jul. 2013, N. Kikuchi (NSMT); 2 §ิ̊̄, Shiraishi-toge, Higashichichibu-mura, Saitama Pref., 20 Jul. 2013, N. Kikuchi (NSMT); 1 ภ, Manazuru, Manazuru-cho, Kanagawa Pref., 30 Jun. 2013, K. Watanabe (KPMNH); 1 q, Tanzawa, Kanagawa Pref., 21 Aug. 1993, K. Kudo (NIAES); 1 q, 1 §, Otone-toge, Hankone, Kanagawa Pref., 27 Jul. 1999, H. Nagase (KPMNH); 1 , Mt Koboyama, Hadano-shi, Kanagawa Pref., 28 Jun. 2008, K. Watanabe (KPMNH); 1 §, Fudago, Kimitsu-shi, Chiba Pref., 18 Jul. 2010, S. Tsuyuki (KPMNH); 1 +, Takekura, Mishima-shi, Shizuoka Pref., 23 Jun.-8 Jul. 2012, A. Ishikawa (KPMNH); 1 q, Inatori, Higashiizu-shi, Shizuoka Pref., 7 Jul. 2009, T. Muraki (KPMNH); 1 Jे, Biwakubo-zawa, Masutomi, Hokuto-shi, Yamanashi Pref., 8 Aug. 2008, K. Watanabe (KPMNH); 1 ․, Tano, Yamato-cho, Koshu-shi, Yamanashi Pref., 5 Aug. 2008, K. Watanabe (KPMNH); 2 q , Maruno-cho, Nirasaki-shi, Yamanashi Pref., 26 Jun.-20 Jul. 2007, K. Hosoda (OMNH); 2 o , , same

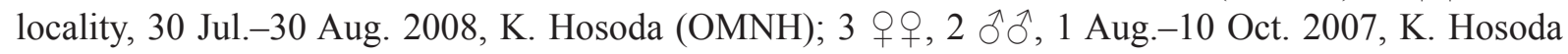
(OMNH); 8 우, 1 §̃, Mt Kariyasuyama, Kaga-cho, Ishikawa Pref., 28 Jun.-19 Jul. 2002, K. Esaki (NIAES); 4 우, same locality, 19-31 Jul. 2002, K. Esaki (NIAES); 3 우, same locality, 18-30 Jul. 2002, K. Esaki (NIAES); 1 q, Kute, Nyukawa-cho, Takayama-shi, Gifu Pref., 4 Aug. 2013, S. Fujie (NSMT); 2 우, Nabedaira-kogen, Takayama-shi, Gifu Pref., 13 Aug. 2013, M. Ito (LC041284 NSMT); 1 q, Sugadaira-kogen, Ueda-shi, nagano Pref., 10 Aug. 2012, D. Kato (LC041277 KPMNH); 1 §ै, same locality, 18 Aug. 2012, D. Kato (KPMNH); 1 , same locality, 8 Aug.-3 Sep. 2014, S. Shimizu (KPMNH); 1 , , Utsukushimatsu, Daimon, Nagawa-cho, Nagano Pref., 26 Aug. 2011, S. Fujie (OMNH); 1 , same locality, 22 Aug. 2012, S. Fujie (LC041280 OMNH); 1 \&, 1 , Ontake-kogen, Outaki-mura, Nagano Pref., 31 Jul. 2013, M. Ito (NIAES); 4 우, Yawata, Asahi-cho, Aichi Pref., 18-28 Jul. 1998, M. Ozawa (MU); 3 우, Sanage, Toyota-shi, Aichi Pref., 8-14 Jul. 2002, M. Kiyota (MU); 2 우, Tanoshiri, Asuke, Aichi Pref., 28 Jun.-5 Jul. 2005, M. Kato (MU); 2 + Mie Pref., 2 Sep. 2012, E. Nishida (OMNH); 2 우, same locality, 7 Aug. 2011, E. Nishida (OMNH);

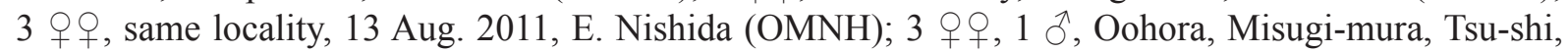
Mie Pref., 24 Jul. 2011, E. Nishida (OMNH); 5 우, Mt Tadosan, Kuwana-shi, Mie Pref., 11 Jun. 2009, A. Kawazoe (EUM); 11 q , same locality, 12 Jul. 2009, A. Kawazoe (EUM); 1 q, Mt Syakagatake, Totsukawa-mura, Nara Pref., 10 Aug. 2013, M. Ito (SEHU); 4 우, same locality, 20 Jul.-11 Aug. 2013, 
S. Fujie and T. Hirooka (SEHU); 1 , Mt Wasamatayama, Kamikitayama-mura, Nara Pref., 26 Jul. 2010, M. Ito (SEHU); 1 +, Mt Odaigaharayama, Kamikitayama-mura, Nara Pref., 8-15 Aug. 2005, A. Kawazoe (OMNH); 1 क , Mt Horaisan, Otsu-shi, Shiga Pref., 16 Aug. 2008, T. Yoshida (SEHU); 1 ð, same locality, 29 Jul. 2008, S. Fujie (SEHU); 1 †, Kamigamo-honzan, Kita-ku, Kyoto Pref., 7 Aug. 2011, M. Ito (OMNH); 1 \&, Hanase-toge, Sakyo-ku, Kyoto-shi, Kyoto Pref., 30 Jul. 2008, N. Nakahama

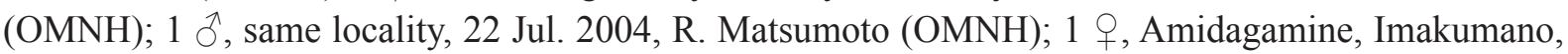
Higashiyama, Kyoto-shi, Kyoto Pref., 4 Jul. 2003, H. Ohishi (OMNH); 1 đ, Baba, Kaizuka-shi, Osaka Pref., 20-30 Jun. 2003, R. Matsumoto (OMNH); 1 +, Saigahara, Minoo-shi, Osaka Pref., 21 Jul.16 Aug. 2006, R. Matsumoto (OMNH); 9 우, Niryou, Takatsuki-shi, Osaka Pref., 30 Jun.-4 Aug. 2013, S. Fujie (OMNH); 1 ô, Oishi-kogen, Wakayama Pref., 15 Jul. 1994, K. Tenma (OMNH); 1 ภ, Mt Koyasan, Koya-cho, Wakayama Pref., 20 Jul. 1999, R. Matsumoto (OMNH); 1 ð, Otousan, Hongu-cho, Wakayama Pref., 13 Jun. 1999, R. Matsumoto (OMNH); 2 ô, Hansarei, Otou-mura, Wakayama Pref.,

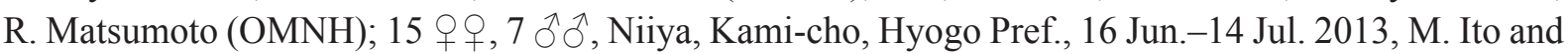
S. Fujie (OMNH); 1 q, same locality, 26 Jun.-18 Jul. 2011 (OMNH); 1 §, same locality, 17 Jul. 2011, S. Fujie (OMNH); 1 q, Akasai-keikoku, Haga-cho, Hyogo Pref., 4 Aug. 2003, R. Matsumoto (OMNH); 2 ô, Yokoiki-keikoku, Mt Hyonosen, Yabu-shi, Hyogo Pref., 13 Jul. 2013, M. Ito (KPMNH); 1 ô, Odanganaru, Mt Hyonosen, Wakasa-cho, Tottori Pref., 6 Aug. 2011, K. Watanabe (KPMNH); 1 ô, Mt Kakezuyama, Geihoku-cho, Hiroshima Pref., 18 Jul. 1998, R. Matsumoto (OMNH); 1 q, Mt

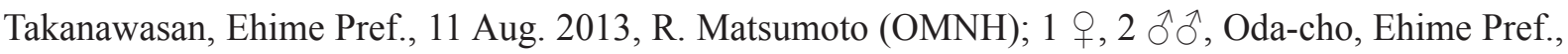
5 Jul. 1997, E. Yamamoto (EUM); 1 ㅇ, same locality, 23 Jul. 1997, E. Yamamoto (EUM); 1 \%, Odamiyama, Namakusadani, Ehime Pref., 16 Jun.1998, E. Yamamoto (OMNH); 2 우, 1 ô, Mt Kanpuzan, Ino-cho, Kochi Pref., 31 Jul. 2006, R. Matsumoto (OMNH); 2 q , , Teragawa, Kochi Pref.,

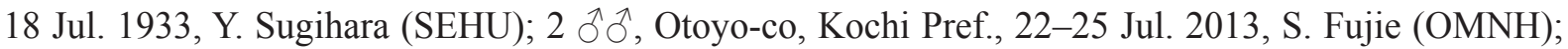
1 ․ Hongawa, Kochi Pref., Jul. 1932, H. Wada (SEHU); 1 , Mt Kuishi, Kochi Pref., 1 Jul. 1931, S. Nakayama (SEHU); 1 q, Ohira, Okawa-mura, Kochi Pref., 21 Aug. 2000, R. Matsumoto (OMNH); 1 , Otaki, Izumi-mura, Kumamoto Pref., 15 Jul. 1999, no data (OMNH); 1 , Gokanosyo, Yatsushiroshi, Kumamoto Pref., 12 Aug. 2011, T. Kawano (SEHU); 1 \&, same locality, 1 Aug. 1976 (SEHU); 1 , Mt Hikosan, Fukuoka Pref., 28-29 Jul. 2007, T. Mita (KPMNH); 1 §̂, Kamishikagawa, Kitagata-machi, Miyazaki Pref., 21 Jul. 2008, K. Katsura (OMNH); 1 §ૈ, Izuhara, Tsushima Is., Nagasaki Pref., 14 Jul. 1992, R. Matsumoto (OMNH); 1 q, Mt Taterasan, Izuhara-cho, Tsushima Is., Nagasaki Pref., 12 Aug. 2001, R. Matsumoto (OMNH); 1 +, Shiratake, Tsushima Is., Nagasaki Pref., 25 Jul. 1994, R. Matsumoto (OMNH); 1 ㅇ, Mitake, Tsushima Is., Nagasaki Pref., 14 Aug. 1993, R. Matsumoto (OMNH); 2 q , Sago, Kamiagata-cho, Tsushima Is., Nagasaki Pref., 5 Jul. 2014, S. Fujie (OMNH); 1 §, Senpironotaki, Yakushima Is., Kagoshima Pref., 8 Jul. 2010, T. Maeda (KPMNH); 1 q, Okawa-falls, Yakushima Is., Kagoshima Pref., 14 Jul. 1994, T. Yamauchi (OMNH); 1 + , Shiratani, Yakushima Is., Kagoshima Pref., 9 Aug.-2 Sep. 2000, T. Murata and K. Nojima (MU); 2 ઈึ, same locality, 21 Jun.-9 Jul. 2000, T. Murata and K. Nojima (MU); 1 \%, Nagata, Yakushima Is., Kagoshima Pref., 28 Jun. 2012, M. Ito

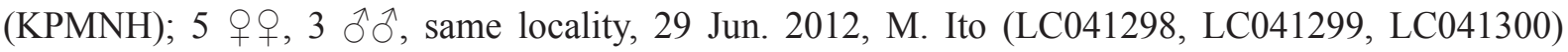

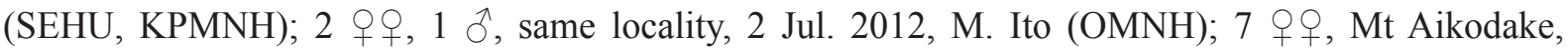
Yakushima Is., Kagoshima Pref., 28 Jun. 2012, M. Ito (KPMNH); 1 q, same locality, 29 Jun. 2012, M. Ito (SEHU); 3 우, same locality, 3 Jul. 2012, M. Ito (OMNH); 1 §, Harumaki, Anbo, Yakushima Is., Kagoshima Pref., 21 Jun. 2012, Y. Kudo (KPMNH).

\section{Differential diagnosis}

As shown in Ito et al. (2015), this species has a distinct geographical gradient in body color across the Japanese Archipelago. For that reason, this species had long been confused with S. apicale (see remarks on $S$. apicale). 


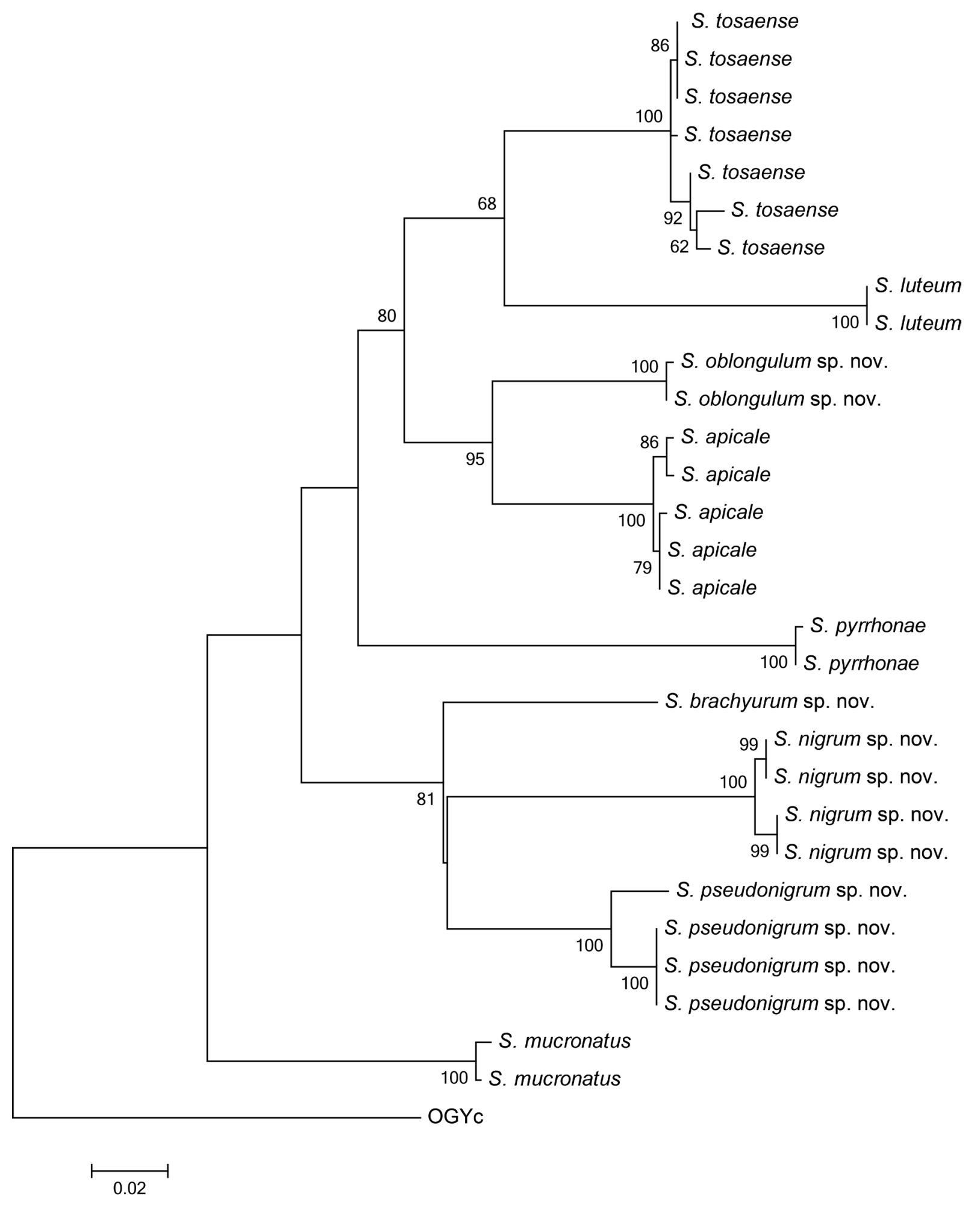

Fig. 9. Maximum-likelihood tree based on mtCOI sequences of Japanese species of Spilopteron and Yamatarotes chishimensis (Uchida, 1929) (OGYc) as an out-group. Bootstrap values $(>50 \%)$ are indicated at the nodes. 


\section{Description}

Female $(\mathrm{n}=181)$

Body LENGTH. 13.0-17.0 mm.

VERTEX. 0.4-0.6 times as long as maximum length of eye in dorsal view. Frons without a depression between eye and antennal socket in frontal view (Fig. 2j-k). Clypeus 0.6-0.7 times as long as wide, with three weak projections (Figs $2 \mathrm{j}-\mathrm{k}, 3 \mathrm{j}$ ). Face $0.6-0.7$ times as long as wide. Length of malar space 0.9-1.2 times as long as basal mandibular width. Ocello-ocular line/lateral ocellar diameter $=1.5-1.8$. Postocellar line/lateral ocellar diameter $=1.1-1.3$. Antenna with 33-35 flagellomeres; first flagellomere 1.3-1.4 times as long as second flagellomere.

Mesosoma. Propleuron densely punctate (Fig. $5 \mathrm{j}-\mathrm{k}$ ). Lateral area of pronotum densely punctate and transversely striate medially (Fig. 5j-k). Collar weakly and sparsely punctate. Mesoscutum densely punctate. Subalar prominence extensively punctate (Fig. $5 j-$ k). Scutellum densely punctate in dorsal view. Postscutellum roundly convex in lateral view (Fig. 5j-k). Metapleuron densely punctate (Fig. 8i). Propodeum indistinctly carinate (Fig. 6j). Area externa densely punctate (Fig. 6j). Area basalis polished (Fig. 6j). Area dentipara, area posteroexterna and area petiolaris confluent with area superomedia (Fig. 6j). Fore wing length $11.5-15.5 \mathrm{~mm}$. Vein $c u$ - $a$ basad of vein $R s \& M$ (Fig. $4 \mathrm{j}-\mathrm{k}$ ). Vein $r s-m$ opposite or basad of vein $2 m-c u$ (Fig. $4 \mathrm{j}-\mathrm{k}$ ). Hind femur 4.0-4.3 times as long as maximum depth in lateral view, without distinct convexity ventrally. Hind tibia 10.6-12.2 times as long as maximum depth in lateral view. First hind tarsomere 2.4-2.5 times as long as second and 2.7-3.2 times as long as longer hind tibial spur.

Metasoma. T1 2.4-2.8 times as long as maximum width, 2.1-2.6 times as long as T2. T2 0.7 times as long as maximum width. T1 weakly and sparsely punctate. T2-T8 weakly and densely punctate. S1 without distinct sharp projection basally. Ovipositor sheath 1.5-1.8 times as long as hind tibia.

Color. Body black to yellowish brown (Fig. 5j-k). Antennal flagellum with a white band. Inner margin of eye, clypeus, basal part of mandible, fore and mid legs, hind legs, and basal part of T1 yellowish brown to black. Fore wing with an apical dark mark extended downwards, and in some individuals with a dark mark below the pterostigma (Fig. 4j-k).

Male ( $n=66$; genitalia, $n=4)$

Similar to female. Body length $12.0-17.0 \mathrm{~mm}$. Head 0.6 times as long as wide in dorsal view. Clypeus $0.7-0.8$ times as long as wide. Face $0.5-0.8$ times as long as wide. Ocello-ocular line/lateral ocellar diameter $=1.2-1.5$. Antenna with 37-43 flagellomeres; first flagellomere 1.2-1.3 times as long as second flagellomere. Hind femur 4.3-4.8 times as long as maximum depth in lateral view. Hind tibia 11.0-13.2 times as long as maximum depth in lateral view. First hind tarsomere 1.9-2.3 times as long as second and 3.3-4.3 times as long as longer hind tibial spur. T1 2.5-2.9 times as long as maximum width, 1.9-2.2 times as long as T2. T2 0.9-1.0 times as long as maximum width. Body invariably yellow with dark stripes.

Subgenital plate hexagonal with basal angle obtuse (Fig. 8i). Paramere short, basal part strongly projecting towards base of subgenital plate (Fig. 7q). Aedeagus gently curved, its penis valve ca 2.0 times as long as basal apodeme (Fig. 7r).

\section{Distribution}

Japan (Hokkaido*, Honshu, Shikoku, Kyushu, Tsushima Is.* and Yakushima Is.) and China. *New records. 


\section{Bionomics}

Host unknown, although in Honshu the first author (M.I.) often observed females ovipositing into coniferous trunks, into which a Cerambycidae (Leptura ochraceofasciata (Motschulsky, 1861)) was also ovipositing. Adults fly from June to August, visiting flowers of Angelica pubescens and Aralia cordata.

\section{Key to the Japanese species of Spilopteron}

1. Hind femur with a distinct convexity ventrally; S1 with a distinct sharp projection basally (see Ito et al. 2012). Ovipositor sheath long (2.7-3.0 times as long as hind tibia)

S. mucronatus Lee, 2008

- Hind femur without a convexity ventrally; S1 without a sharp projection basally. Ovipositor sheath short to long (less than 2.6 times as long as hind tibia)

2. Postscutellum roundly convex in lateral view (Fig. 5b, d, g, j-k). Body with yellow markings or entirely reddish yellow (Fig. 5b, d); males never with a white band on antennal flagellum (Fig. 1h); body relatively large $(10-17 \mathrm{~mm})$

- Postscutellum flat in lateral view (Fig. 5a, c, e, f, h-i). Body entirely black or with yellow markings (Fig. 5a, c); males sometimes with a white band on antennal flagellum (Fig. 1b); body relatively small $(6-13 \mathrm{~mm})$

3. Body entirely reddish yellow (Fig. 5d); fore wing with only an infuscate marking apically (Fig. 4d)

S. luteum (Uchida, 1934)

- Body at least with black parts (Fig. 5b, k); fore wing with one or two distinct dark markings (Fig. 4b, k)

4. Depression between eye and antennal socket absent (Fig. 2j-k); clypeus with three weak projections (Fig. 2j-k). Body black with yellow markings or yellow with black markings (Fig. 5j-k); fore wing sometimes with a black spot below the pterostigma (Fig. 4k)

S. tosaense (Uchida, 1934)

- Depression between eye and antennal socket present (Fig. 2b, g); clypeus with two strong lateral projections (Fig. 2b, g). Body black with yellow markings (Fig. 5b, g); fore wing never with a black spot below the pterostigma (Fig. $4 \mathrm{~b}, \mathrm{~g}$ )

5. Apical black marking of fore wing extending downwards (Fig. 4g); T1 slender (3.5-4.3 times as long as wide); ovipositor sheath long (2.3-2.6 times as long as hind tibia)

S. oblongulum sp. nov.

- Apical black marking of fore wing rounded, not extending downwards (Fig. 4b); T1 broad (2.2-3.3 times as long as wide); ovipositor sheath short (1.8 times as long as hind tibia) S. apicale (Matsumura, 1912)

6. Fore wing with only an infuscate apical portion (Fig. 4c, h) ..... 7

- Fore wing with a distinct black marking apically (Fig. 4a, f, i) 8

7. Area superomedia of propodeum rounded (Fig. 6c); ovipositor sheath short (1.9 times as long as hind tibia) (Fig. 1c). Antennal flagellum with a white band in female and male (Fig. 1d)

S. brachyurum sp. nov.

- Area superomedia of propodeum squared (Fig. 6h); ovipositor sheath long (2.0-2.3 times as long as hind tibia). Antennal flagellum with a white band in female but without it in male (Fig. 1f, j) 
8. Malar space long (length of malar space 1.3-1.4 times as long as basal mandibular width); apex of metasoma yellowish white (Fig. 1a-b) S. albiventre sp. nov.

- Malar space short (length of malar space 0.9-1.2 times as long as basal mandibular width); apex of metasoma black (Fig. 1e-f)

9. Apical black marking of fore wing rounded, not extending downwards (Fig. 1i); apical margin of clypeus turned up in lateral view. Antennal flagellum with a white band in female and male .....

S. pyrrhonae Kusigemati, 1981

- Apical black marking of fore wing extending downwards (Fig. 1f); apical margin of clypeus flattened. Antennal flagellum with a white band in female but without it in male (Fig. 1f)

S. nigrum sp. nov.

\section{Discussion}

We recognize ten species of Spilopteron from Japan, of which six occur in Hokkaido, nine in Honshu, five in Shikoku, four in Kyushu and two in the Nansei Islands (Yakushima Is., Amami-oshima Is.). In other countries, this genus is known from the U.S.A. (four species), Canada (three species), Far East Russia (one species), mainland China (22 species), Korea (one species), and Taiwan (two species) (Yu et al. 2012; Ito et al. 2012). Therefore, this genus seems to have its center of diversity in the midlatitude area of East Asia.

In terms of body color, female $S$. tosaense are known to show an increasing melanism from south to north in the Japanese Archipelago (Ito et al. 2015). Also, S. luteum, distributed in Taiwan and southern Japan, has an entirely yellowish body color, although most Japanese species are dark. Similarly, many Chinese species of Spilopteron in southern areas have a yellowish body color (Wang 2004). In another case from ichneumonids, species of Apechthis Förster, 1869 show two conspicuous body color patterns: black or yellow (Watanabe \& Takasuka 2013). The black species are mainly distributed in the Holarctic and Himalayan regions, but the yellow species are mainly in the Oriental and Neotropical regions. Therefore, such geographical color variation within or among species, along a latitudinal gradient or related to ecology (open or closed environment), seems not to be uncommon in ichneumonid wasps.

\section{Acknowledgements}

We thank K. Konishi (Ehime University), T. Yoshida (SEHU), K. Watanabe (KPMNH), R. Matsumoto $(\mathrm{OMNH})$, S. Shimizu (Tsukuba University) and S. Fujie (Osaka) for kindly offering materials, K. Kusigemati, K. Tsuda, Y. Sakamaki (KU), S. Yoshimatsu (NIAES), A. Shinohara (NSMT) and M. Ohara (SEHU) for their kind support in the investigation of their collections, M. Takeda and K. Sakamoto (Kobe University) for their kind permission to use their laboratory, and K. Ikeda (Kobe University) for his help in using the electron microscope. This research was in part supported by the Sasakawa Scientific Research Grant from the Japan Science Society to M.I. and JSPS KAKENHI (Grant Number 25292034) to K.M.

\section{References}

Champlain A.B. 1922. Records of Hymenopterous parasites in Pennsylvania. Psyche 29: 95-100. https:// doi.org/10.1155/1922/98647

Chiu S.C. 1971. The Taiwan Acaenitinae (Hymenoptera: Ichneumonidae). Bulletin of the Taiwan Agricultural Research Institute 29: 1-26.

Cushman R.A. 1933. H. Sauter's Formosa-collection: subfamily Ichneumoninae (Pimplinae of Ashmead). Insecta Matsumurana 8: 1-50.

Cushman R.A. \& Rohwer S.A. 1920. The North American Ichneumon-flies of the tribe Acoenitini. Proceedings of the United States National Museum 57: 503-523. https://doi.org/10.5479/si.00963801.57$\underline{2320.503}$ 
Eady R.D. 1968. Some illustrations of microsculpture in the Hymenoptera. Proceedings of the Royal Entomological Society of London 43: 66-72. https://doi.org/10.1111/j.1365-3032.1968.tb01029.x

Folmer O., Black M., Hoeh W., Lutz R. \& Vrijenhoek R. 1994. DNA primers for amplification of mitochondrial cytochrome c oxidase subunit I from diverse metazoan invertebrates. Molecular Marine Biology and Biotechnology 3: 294-297.

Gauld I.D. 1991. The Ichneumonidae of Costa Rica, 1. Memoirs of the American Entomological Institute 47: $1-589$.

Gauld I.D. 2002. The Ichneumonidae of Costa Rica, 4. Memoirs of American Entomological Institute 66: $1-768$.

Ito M. \& Maeto K. 2014. Revision of the genus Yamatarotes Uchida (Hymenoptera: Ichneumonidae: Acaenitinae) from Japan, based on morphological and molecular evidence. Japanese Journal of Systematic Entomology 20: 107-113.

Ito M., Watanabe K. \& Maeto K. 2012. Spilopteron luteum Uchida and S. mucronatus Lee (Hymenoptera, Ichneumonidae, Acaenitinae) new to Japan. Japanese Journal of Systematic Entomology 18: 443-446.

Ito M., Watanabe K. \& Maeto K. 2015. Molecular evidence resolving the confusion of two species of Spilopteron (Hymenoptera: Ichneumonidae) caused by marked geographical colour variation. European Journal of Entomology 112: 543-556. https://doi.org/10.14411/eje.2015.068

Kimura M. 1980. A simple method for estimating evolutionary rate of base substitution through comparative studies of nucleotide sequence. Journal of Molecular Evolution 16: 111-120. https://doi. org/10.1007/BF01731581

Kusigemati K. 1981. Japanese species of the genus Spilopteron with description of a new species (Hymenoptera, Ichneumonidae). Memoirs of the Faculty of Agriculture, Kagoshima University 17: $117-125$.

Lee J.W., Jeong J.C. \& Lee S.M. 2008. First record of the genus Spilopteron (Hymenoptera: Ichneumonidae: Acaenitinae) from Korea with description of a new species. Korean Journal of Systematic Zoology 24 (3): 275-279.

Matsumura S. 1912. Thousand Insects of Japan. Supplement IV. Keiseisha, Tokyo.

Sakurai A., Hamanishi H., Maeto K. \& Naito C. 2009. Molecular biogeography of two sibling species of the sawfly genus Macrophya (Hymenoptera: Tenthredinidae) in Japan. Zoological Science 26: 325-329. https://doi.org/10.2108/zsj.26.325

Shaw M.R. \& Wahl D.B. 1989. The biology, eggs and larvae of Acaenitus dubitator (Panzer) (Hymenoptera: Ichneumonidae: Acaenitinae). Systematic Entomology 14: 117-125. https://doi. org/10.1111/j.1365-3113.1989.tb00269.x

Sheng M.L. \& Sun S.P. 2010. Parasitic ichneumonids on woodborers in China (Hymenoptera: Ichneumonidae). Science Press, Beijing [in Chinese with English summary].

Sonan J. 1936. Description of and notes on some Pimplinae in Formosa (Hym. Ichneumonidae). Transactions of the Natural History Society of Formosa, Taihoku 26 (153): 249-257.

Stigenberg J., Vikberg V. \& Belokobylskij S.A. 2011. Meteorus acerbiavorus sp. nov. (Hymenoptera, Braconidae), a gregarious parasitoid of Acerbia alpina (Quensel) (Lepidoptera, Arctiidae) in North Finland. Journal of Natural History 45: 1275-1294. https://doi.org/10.1080/00222933.2011.552807

Tamura K., Stecher G., Peterson D., Filipski A. \& Kumar S. 2013. MEGA6: Molecular evolutionary genetics analysis version 6.0. Molecular Biology and Evolution 30: 2725-2729. https://doi.org/10.1093/ $\underline{\text { molbev/mst197 }}$

Townes H.K. 1944. A catalogue and reclassification of the Nearctic Ichneumonidae (Hymenoptera). Part I. The subfamilies Ichneumoninae, Tryphoninae, Cryptinae, Phaeogeninae and Lissonotinae. Memoirs 
of the American Entomological Society 11: 1-477. Available from http://www.biodiversitylibrary.org/ page/38650196\#page/13/mode/1up [accessed 12 Jul. 2017].

Townes H. 1971. The genera of Ichneumonidae, Part 4. Memoirs of the American Entomological Institute 17: $1-372$.

Townes H.K., Townes M., Walley G.S. \& Townes G. 1960. Ichneumon-flies of America north of Mexico: 2. Subfamily Ephialtinae, Xoridinae, Acaenitinae. United States National Museum Bulletin 216: 1-676.

Townes H., Townes M. \& Gupta V.K. 1961. A catalogue and reclassification of the Indo-Australian lchneumonidae. Memoirs of the American Entomological Institute 1: 1-522.

Townes H., Momoi S. \& Townes M. 1965. A catalogue and reclassification of the eastern Palearctic Ichneumonidae. Memoirs of the American Entomological Institute 5: 1-661.

Uchida T. 1928. Dritter Beitrag zur Ichneumoniden-Fauna Japans. Journal of the Faculty of Agriculture, Hokkaido Imperial University 25: 1-115.

Uchida T. 1930. Beschreibungen der neuen echten Schlupfwespen aus Japan, Korea und Formosa. Insecta Matsumurana 4: 121-132.

Uchida T. 1932. Beitrage zur Kenntnis der japanischen Ichneumoniden. Insecta Matsumurana 6: 145168.

Uchida T. 1934. Beiträge zur Systematik der Tribus Acoenitini Japans (Hym. Ichneum. Pimplinae). Insecta matsumurana 9: 41-54.

Wahl D.W. \& Gauld I.D. 1998. The cladistics and higher classification of the Pimpliforms (Hymenoptera: Ichneumonidae). Systematic Entomology 23: 265-298.

Wang S.F. \& Li W.Z. 2004. The genus Spilopteron Townes (Hymenoptera: Ichneumonidae) in China. Oriental Insects 38: 63-75.

Watanabe K. \& Takasuka K. 2013. Description of Apechthis cantika sp. n. from Sulawesi Is., Indonesia with redescription of the holotype of A. taiwana Uchida (Hymenoptera, Ichneumonidae, Pimplinae). Journal of Hymenoptera Research 31: 105-117.

Yu D.S., Achterberg K. van \& Horstmann K. 2005. World Ichneumonoidea 2004. Taxonomy, biology, morphology and distribution. Taxapad $\AA$, Vancouver [CD-ROM].

Yu D.S., Achterberg K. van \& Horstmann K. 2012. World Ichneumonoidea 2011. Taxonomy, biology, morphology and distribution. Taxapad®, Vancouver [Flash drive].

Manuscript received: 8 June 2016

Manuscript accepted: 17 December 2016

Published on: 5 October 2017

Topic editor: Gavin Broad

Desk editor: Chloe Chester

Printed versions of all papers are also deposited in the libraries of the institutes that are members of the EJT consortium: Muséum national d'Histoire naturelle, Paris, France; Botanic Garden Meise, Belgium; Royal Museum for Central Africa, Tervuren, Belgium; Natural History Museum, London, United Kingdom; Royal Belgian Institute of Natural Sciences, Brussels, Belgium; Natural History Museum of Denmark, Copenhagen, Denmark; Naturalis Biodiversity Center, Leiden, the Netherlands; Museo Nacional de Ciencias Naturales-CSIC, Madrid, Spain; Real Jardín Botánico de Madrid CSIC, Spain. 